\title{
Probing the Effects of Strong Electromagnetic Fields with Charge-Dependent Directed Flow in Pb-Pb Collisions at the LHC
}

\author{
S. Acharya et al. ${ }^{*}$ \\ (A Large Ion Collider Experiment Collaboration)
}

(Received 25 November 2019; revised 22 April 2020; accepted 19 May 2020; published 6 July 2020)

\begin{abstract}
The first measurement at the LHC of charge-dependent directed flow $\left(v_{1}\right)$ relative to the spectator plane is presented for $\mathrm{Pb}-\mathrm{Pb}$ collisions at $\sqrt{s_{\mathrm{NN}}}=5.02 \mathrm{TeV}$. Results are reported for charged hadrons and $\mathrm{D}^{0}$ mesons for the transverse momentum intervals $p_{\mathrm{T}}>0.2 \mathrm{GeV} / c$ and $3<p_{\mathrm{T}}<6 \mathrm{GeV} / c$ in the $5 \%-40 \%$ and $10 \%-40 \%$ centrality classes, respectively. The difference between the positively and negatively charged hadron $v_{1}$ has a positive slope as a function of pseudorapidity $\eta, \mathrm{d} \Delta v_{1} / \mathrm{d} \eta=$ $[1.68 \pm 0.49$ (stat) \pm 0.41 (syst) $] \times 10^{-4}$. The same measurement for $\mathrm{D}^{0}$ and $\overline{\mathrm{D}}^{0}$ mesons yields a positive value $\mathrm{d} \Delta v_{1} / \mathrm{d} \eta=[4.9 \pm 1.7$ (stat) $\pm 0.6($ syst $)] \times 10^{-1}$, which is about 3 orders of magnitude larger than the one of the charged hadrons. These measurements can provide new insights into the effects of the strong electromagnetic field and the initial tilt of matter created in noncentral heavy ion collisions on the dynamics of light ( $\mathrm{u}, \mathrm{d}$, and s) and heavy (c) quarks. The large difference between the observed $\Delta v_{1}$ of charged hadrons and $\mathrm{D}^{0}$ mesons may reflect different sensitivity of the charm and light quarks to the early time dynamics of a heavy ion collision. These observations challenge some recent theoretical calculations, which predicted a negative and an order of magnitude smaller value of $\mathrm{d} \Delta v_{1} / \mathrm{d} \eta$ for both light flavor and charmed hadrons.
\end{abstract}

DOI: 10.1103/PhysRevLett.125.022301

Quantum chromodynamic (QCD) calculations on the lattice [1-6] predict at high temperatures the existence of a deconfined state of quarks and gluons, known as the quarkgluon plasma (QGP). Characterizing the QGP properties is among the main goals of the experimental program with ultrarelativistic heavy ion collisions at the Large Hadron Collider (LHC). Measurements of the anisotropic transverse flow [7-11], quantified by the second $\left(v_{2}\right)$ and higher order $(n>2)$ harmonic coefficients $v_{n}$, allow one to characterize the different phases of a heavy ion collision and constrain the properties of the QGP [12-16].

The directed flow, $v_{1}$, has a special role due to its sensitivity to the three-dimensional spatial profile of the initial conditions and the preequilibrium early time dynamics in the evolution of the collision. The space momentum correlations in particle production from a longitudinally tilted source result in a nonzero $v_{1}$. The tilt arises from the asymmetries in the number of forward and backward moving participant nucleons at different positions in the transverse plane [17-19]. The directed flow of charged

\footnotetext{
*Full author list given at the end of the article.

Published by the American Physical Society under the terms of the Creative Commons Attribution 4.0 International license. Further distribution of this work must maintain attribution to the author(s) and the published article's title, journal citation, and DOI.
}

hadrons at the LHC [20] has significantly smaller magnitude compared to that at lower relativistic heavy ion collider (RHIC) energies [21], which can be interpreted as a smaller initial tilt at the LHC [22-24].

Charm quarks are produced early in the collision via hard scattering processes. Their emission region is not tilted in the longitudinal direction [19] unlike the one of light quarks, which are predominantly produced in soft processes at later stages of the collision [18,25]. Consequently, the region of charm quark production in the transverse plane is shifted with respect to that of light quarks and gluons, resulting in an enhanced dipole asymmetry in the charm quark distribution [19]. During the system expansion, charm quarks would be dragged by the flow of the light quarks in the transverse direction of the shift, which is predicted to result in a larger $v_{1}$ of charm hadrons compared to light flavor hadrons $[19,26]$. Consequently, the measurements of the charge-integrated directed flow of hadrons containing light (u, d, and s) and heavy (c) quarks together with their difference in magnitude are of great interest and allow one to probe the three-dimensional space-time evolution of the produced matter.

Heavy ion collisions are also characterized by extremely strong electromagnetic fields primarily induced by spectator protons, which do not undergo inelastic collisions. There is strong interest in characterizing the time evolution of these fields, which are estimated to reach $10^{18}-10^{19}$ Gauss in the early stages $(<0.5 \mathrm{fm})$ of $\mathrm{Pb}-\mathrm{Pb}$ collisions at 
LHC energies [27,28]. Phenomena predicted to occur in the presence of this strong electromagnetic field include the chiral magnetic effect (CME), which is driven by the generation of an electric current along the magnetic field in a medium with chiral imbalance [29-32]. While experimental results for charge-dependent correlations are in qualitative agreement with theoretical expectations for the CME [33-35], possible background contributions, such as effects of local charge conservation coupled with the anisotropic flow, prevent their unambiguous interpretation [36] and have led to upper limits on the CME at LHC energies. Thus, it is fundamental to use other observables with direct sensitivity to the electromagnetic fields in order to constrain their magnitudes and time evolution in heavy ion collisions.

The charge dependence of the produced particle directed flow relative to the spectator plane is directly sensitive to the presence of electromagnetic fields. The spectator plane is defined by the deflection direction of the collision spectators. On average, its orientation is perpendicular to the direction of the magnetic field generated by the positively charged spectators. The charge dependence of $v_{1}$ comes from two competing effects acting in opposite directions: the Lorentz force and the Coulomb force, both induced by the rapidly decreasing magnetic field. In an electrically conducting plasma, the induced charged currents might slow down the decay of the magnetic field [27]. The measurement of charge-dependent $v_{1}$ can therefore constrain the QGP electric conductivity.

First estimates of these effects on the $v_{1}$ of charged particles were presented in $[37,38]$. Using the electric conductivity from lattice QCD calculations $[39,40]$, the difference between the $v_{1}$ of positively and negatively charged pions, $\Delta v_{1}(\pi)=v_{1}\left(\pi^{+}\right)-v_{1}\left(\pi^{-}\right)$, in Pb-Pb collisions at $\sqrt{s_{\mathrm{NN}}}=2.76 \mathrm{TeV}$ was estimated to be not larger than $10^{-5}$ for $|\eta|<1$ [38]. Charm quarks, which are produced in the early stages when the magnetic field is maximal, should be more strongly affected by the electromagnetic fields than light quarks [26,41]. The difference $\Delta v_{1}$ between the $v_{1}$ of $\mathrm{D}^{0}(\mathrm{c} \bar{u})$ and $\overline{\mathrm{D}}^{0}(\overline{\mathrm{c} u})$ mesons should therefore provide better sensitivity to this initial magnetic field. A calculation of $v_{1}$ of charmed mesons in the rapidity interval $|y|<1$ gives a value $\Delta v_{1}(D) \sim 10^{-2}$ [41], which is 3 orders of magnitude larger than the one expected for pions. Recently, STAR Collaboration published a measurement of directed flow of $\mathrm{D}^{0}$ and $\overline{\mathrm{D}}^{0}$ mesons in $10 \%-$ $80 \%$ central $\mathrm{Au}-\mathrm{Au}$ collisions at $\sqrt{s_{\mathrm{NN}}}=200 \mathrm{GeV}$ [42]. The slope of $v_{1}$ of $\mathrm{D}^{0}$ and $\overline{\mathrm{D}}^{0}$ mesons as a function of rapidity is negative and about a factor of 25 times larger than that of charged kaons.

This Letter reports the first measurements at the LHC of the charge dependence of $v_{1}$ relative to the spectator plane for charged hadrons and $\mathrm{D}^{0}$ mesons as a function of pseudorapidity in midcentral $\mathrm{Pb}-\mathrm{Pb}$ collisions at $\sqrt{s_{\mathrm{NN}}}=5.02 \mathrm{TeV}$.
About $23(19) \times 10^{6} \mathrm{~Pb}-\mathrm{Pb}$ collisions in the $5 \%-40 \%$ $(10 \%-40 \%)$ centrality interval are used for the charged hadron ( $\mathrm{D}^{0}$ and $\overline{\mathrm{D}}^{0}$ meson) $v_{1}$ measurements. Only events with a primary vertex reconstructed within $\pm 10 \mathrm{~cm}$ from the detector center along the beam direction are analyzed. Two forward scintillator arrays (V0A and V0C) [43] are used to determine the collision centrality. For the most central $(0 \%-5 \%)$ collisions, the small number of spectators prevents an accurate reconstruction of their deflection. In the $5 \%-10 \%$ centrality interval, the large combinatorial background does not allow the measurement of the $\mathrm{D}^{0}$ and $\overline{\mathrm{D}}^{0} v_{1}$.

The deflection direction of the collision spectators is reconstructed from spectator neutrons detected using two zero degree calorimeters (ZDCs) [44,45]. The ZDCs have a $2 \times 2$ segmentation in the plane transverse to the beam direction and are installed at $112.5 \mathrm{~m}$ distance from the detector center on both sides of the interaction point, covering the "projectile" $(\eta>8.78)$ and the "target" $(\eta<-8.78)$ spectator regions. For each ZDC, a flow vector is constructed following the procedure described in [20]:

$$
\mathbf{Q}^{t, p} \equiv\left(\mathrm{Q}_{x}^{t, p}, \mathrm{Q}_{y}^{t, p}\right)=\sum_{i=1}^{4} \mathbf{n}_{i} E_{i}^{t, p} / \sum_{i=1}^{4} E_{i}^{t, p},
$$

where $p$ and $t$ denote the ZDC on the projectile and target side, $E_{i}$ is the measured signal, and $\mathbf{n}_{i}=\left(x_{i}, y_{i}\right)$ are the coordinates of the center of the $i$ th ZDC segment.

The deflection direction of the spectator neutrons is estimated event by event with the $\mathbf{Q}^{t, p}$ vectors corrected for the run-dependent variation of the LHC beam crossing position [46]. In midcentral collisions, this deflection direction is strongly correlated with the magnetic field orientation. The deflection is expected to be opposite (anticorrelated) for the projectile and the target sides, i.e., $\left\langle\mathrm{Q}_{x}^{p} \mathrm{Q}_{x}^{t}\right\rangle=\left\langle\mathrm{Q}_{y}^{p} \mathrm{Q}_{y}^{t}\right\rangle<0$ and $\left\langle\mathrm{Q}_{y}^{p} \mathrm{Q}_{x}^{t}\right\rangle$ and $\left\langle\mathrm{Q}_{x}^{p} \mathrm{Q}_{y}^{t}\right\rangle=0$. A deviation from these expectations, mostly for peripheral collisions with centrality above $40 \%$, is observed even after applying the flow vector correction. These residual variations are used in the estimation of the systematic uncertainty as described in [20] and discussed below.

The directed flow is measured using the scalar product method [47] as follows:

$$
v_{1}^{t, p}=\frac{\left\langle\mathbf{u} \mathbf{Q}^{t, p}\right\rangle}{\sqrt{\left|\left\langle\mathbf{Q}^{t} \mathbf{Q}^{p}\right\rangle\right|}}=\frac{\left\langle\mathrm{u}_{x} \mathrm{Q}_{x}^{t, p}+\mathrm{u}_{y} \mathrm{Q}_{y}^{t, p}\right\rangle}{\sqrt{\left|\left\langle\mathrm{Q}_{x}^{t} \mathrm{Q}_{x}^{p}+\mathrm{Q}_{y}^{t} \mathrm{Q}_{y}^{p}\right\rangle\right|}},
$$

where $\mathbf{u}=(\cos \varphi, \sin \varphi)$ is the unit flow vector of the charged hadron or $\mathrm{D}^{0}$ meson candidate with azimuthal angle $\varphi$. The directed flow is calculated as $v_{1}=\left(v_{1}^{p}-v_{1}^{t}\right) / 2$. The sign of $v_{1}$ is defined relative to the deflection of the projectile spectators, corresponding to the rapidity odd component of the $v_{1}$ discussed in [20]. The measurement of $v_{1}$ using 
spectators does not require any treatment of the momentum conservation unlike the measurements based on correlations between particles produced at midrapidity [48]. This is justified by the observation of a vanishing relative momentum shift along the spectator plane at $\eta=0$ [20].

The charged hadron $v_{1}$ is measured from tracks reconstructed with the Inner Tracking System (ITS) [49] and the time projection chamber (TPC) [50] and selected requiring $p_{\mathrm{T}}>0.2 \mathrm{GeV} / c,|\eta|<0.8$, at least 70 (out of a maximum of 159) TPC space points and $\chi^{2} / \mathrm{ndf}<2$ for the momentum fit in the TPC. In order to reduce the contamination from secondary particles, only tracks with a maximum distance of closest approach (DCA) to the reconstructed primary vertex in both the transverse $\left(\mathrm{DCA}_{x y}<2.4 \mathrm{~cm}\right)$ and the longitudinal direction $\left(\mathrm{DCA}_{z}<3.2 \mathrm{~cm}\right)$ are accepted.

The $\mathrm{D}^{0}$ and $\overline{\mathrm{D}}^{0}$ mesons are reconstructed using the decay channel $\mathrm{D}^{0} \rightarrow K^{-} \pi^{+}$and its charge conjugate for $3<p_{\mathrm{T}}<6 \mathrm{GeV} / c$. Pions and kaons are reconstructed in the TPC and ITS detectors. Tracks are selected requiring $|\eta|<0.8, p_{\mathrm{T}}>0.4 \mathrm{GeV} / c$, at least 70 hits in TPC, and at least two hits (out of a maximum of six) in the ITS, out of which at least one has to be in the two innermost layers. Particle identification is based on measurements of the specific ionization energy loss $\mathrm{d} E / \mathrm{d} x$ in the TPC and the flight time from the interaction point to the time of flight (TOF) detector [51]. The charge of the identified pions and kaons allows one to distinguish between the $\mathrm{D}^{0} \rightarrow \mathrm{K}^{-} \pi^{+}$ and $\overline{\mathrm{D}}^{0} \rightarrow \mathrm{K}^{+} \pi^{-}$candidates. Geometrical selections on the displaced decay vertex topology are applied to reduce the combinatorial background [52].

The $v_{1}^{\mathrm{D}}$ is extracted separately for $\mathrm{D}^{0}$ and $\overline{\mathrm{D}}^{0}$ mesons via a simultaneous fit to the number $N(M)$ of $K^{\mp} \pi^{ \pm}$pairs and their $v_{1}(M)$ as a function of the invariant mass, $M$ :

$N(M)=N_{D}(M)+N_{\mathrm{bg}}(M)$,

$v_{1}(M)=\left[v_{1}^{D} N_{D}(M)+v_{1}^{\mathrm{bg}}(M) N_{\mathrm{bg}}(M)\right] /\left[N_{D}(M)+N_{\mathrm{bg}}(M)\right]$.

An example of the simultaneous fit is shown in Fig. 1. The invariant mass distribution is fitted with the sum of a Gaussian function $N_{D}(M)$ for the $\mathrm{D}^{0}$ and $\overline{\mathrm{D}}^{0}$ signal and an exponential function $N_{\mathrm{bg}}(M)$ for the background. The invariant mass dependence of the directed flow of background candidates $v_{1}^{\mathrm{bg}}(M)$ is parameterized by a linear function.

Candidates that satisfy both the $\mathrm{K}^{-} \pi^{+}$and $\mathrm{K}^{+} \pi^{-}$ hypotheses (reflected kinematics) and therefore cannot be tagged uniquely as $\mathrm{D}^{0}$ or $\overline{\mathrm{D}}^{0}$ are rejected. This removes about $35 \%$ of the signal and increases the signal to background ratio by about $30 \%-40 \%$, with a net result of a negligible reduction of the statistical significance of the $\mathrm{D}^{0}$ and $\overline{\mathrm{D}}^{0}$ yield. The extracted $v_{1}^{\mathrm{D}}$ includes contributions from both prompt $\mathrm{D}^{0}$ mesons and feed-down $\mathrm{D}^{0}$ mesons

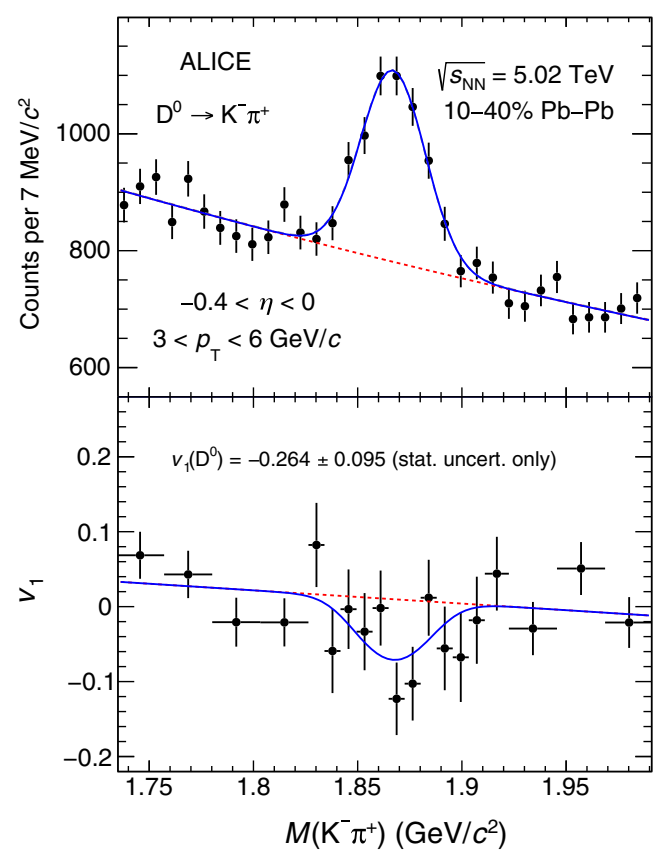

FIG. 1. Illustration of the $\mathrm{D}^{0}$ meson $v_{1}$ extraction procedure via a simultaneous fit to the candidate invariant mass distribution (upper panel) and $v_{1}$ (lower panel) for $3<p_{\mathrm{T}}<6 \mathrm{GeV} / c$ and $-0.4<\eta<0$ in the $10 \%-40 \%$ centrality interval. The blue solid lines correspond to the combined signal and background fit functions, while the red dashed lines represent the background contribution.

from beauty hadron decays. The fraction of prompt $\mathrm{D}^{0}$ meson is about $85 \%$ for the analyzed centrality class and $p_{\mathrm{T}}$ interval [53].

Common sources of systematic uncertainty between charged hadrons and D mesons are related to the resolution of the spectator plane and to the dependence on the ALICE magnet polarity. The absolute systematic uncertainty related to the residual asymmetry in the spectator plane estimation is given by the difference between the $v_{1}$ obtained separately from $\left\langle\mathrm{u}_{x} \mathrm{Q}_{x}\right\rangle$ and $\left\langle\mathrm{u}_{y} \mathrm{Q}_{y}\right\rangle$ correlations with the ZDCs in Eq. (2). It is about $3.5 \times 10^{-5}\left(2 \times 10^{-2}\right)$ for charged hadrons ( $\mathrm{D}^{0}$ and $\overline{\mathrm{D}}^{0}$ mesons). Effects related to track reconstruction and geometrical alignment of the detectors, which could influence positive and negative tracks differently, are estimated by comparing the $v_{1}$ results obtained using data taken with opposite magnet polarity. This comparison also probes the bias in the spectator plane estimation due to the nonzero beam crossing angle in the vertical plane, which had opposite values $( \pm 60 \mu \mathrm{rad})$ for the opposite magnet polarities. The absolute difference between the $v_{1}$ values obtained with the two field polarities is $2.5 \times 10^{-5}\left(2 \times 10^{-2}\right)$ for charged hadrons $\left(\mathrm{D}^{0}\right.$ and $\left.\overline{\mathrm{D}}^{0}\right)$. These systematic uncertainties are correlated in pseudorapidity for charged hadrons, while for $\mathrm{D}^{0}$ and $\overline{\mathrm{D}}^{0}$ mesons no significant correlation, beyond statistical uncertainties, is observed. 
For charged hadrons, the track quality selections are varied and an absolute systematic uncertainty of $2.5 \times 10^{-5}$ is assigned. The contribution from secondaries is varied by changing the maximum $\mathrm{DCA}_{x y}$, which resulted in a negligible variation of $v_{1}$. The contamination due to TPC tracks originating from pileup collisions during the readout time of the TPC is estimated by varying the selections on the correlations between the event multiplicity (centrality) estimated with detectors with different readout times. The resulting systematic uncertainty is $10^{-5}$ for the charged hadrons. No systematic uncertainty is assigned for $\mathrm{D}^{0}$ and $\overline{\mathrm{D}}^{0}$ because the topological selections effectively remove tracks from pileup. The uncertainty due to the $\mathrm{D}^{0}$ and $\overline{\mathrm{D}}^{0}$ signal extraction is estimated by varying (i) the fit functions in Eqs. (3) and (4) for $N(M)$ and $v_{1}(M)$, (ii) fixing the Gaussian width and mean to the values extracted from Monte Carlo simulations, and (iii) varying the invariant mass fit range. The absolute systematic uncertainty assigned to $v_{1}$ due to the $\mathrm{D}^{0}$ and $\overline{\mathrm{D}}^{0}$ yield extraction is $2 \times 10^{-2}$. The possible bias due to the $p_{\mathrm{T}^{-}}$-dependent efficiency in the $\mathrm{D}^{0}$ and $\overline{\mathrm{D}}^{0} v_{1}$ analysis is tested by reweighting both signal and background with the inverse value of the signal reconstruction efficiency as a function of $p_{\mathrm{T}}$. The assigned absolute systematic uncertainty is $10^{-2}$.

The total systematic uncertainty on $v_{1}$ is obtained by adding in quadrature the contributions described above.
In the calculation of $\Delta v_{1}(D)$, all individual systematic uncertainties are propagated as fully uncorrelated between $\mathrm{D}^{0}$ and $\overline{\mathrm{D}}^{0}$. For charged hadrons, the systematic uncertainties due to the asymmetry in the spectator plane estimation and the magnet polarity are correlated between positive and negative tracks and largely cancel in $\Delta v_{1}(h)$.

The pseudorapidity dependence of the directed flow of positively and negatively charged hadrons for the 5\%-40\% centrality class in $\mathrm{Pb}-\mathrm{Pb}$ collisions at $\sqrt{s_{\mathrm{NN}}}=5.02 \mathrm{TeV}$ is shown in the upper left panel of Fig. 2. The negative slope of $v_{1}$ is usually attributed to the effect of the initial tilt [18] or rotation [25] of the particle-emitting source. The chargeintegrated $v_{1}$ at $\sqrt{s_{\mathrm{NN}}}=5.02 \mathrm{TeV}$ agrees within uncertainties with the results at $\sqrt{s_{\mathrm{NN}}}=2.76 \mathrm{TeV}$ [20].

The difference $\Delta v_{1}(h)$ between the $v_{1}$ of positively and negatively charged hadrons as a function of pseudorapidity is shown in the lower left panel of Fig. 2. The rapidity slope $\mathrm{d} \Delta v_{1} / \mathrm{d} \eta$, extracted with a linear fit (constrained to $v_{1}=0$ at $\eta=0)$, is $\mathrm{d} \Delta v_{1} / \mathrm{d} \eta=[1.68 \pm 0.49$ (stat) \pm 0.41 (syst) $] \times$ $10^{-4}$ with a significance of $2.6 \sigma$ for having a positive value. The $\mathrm{d} \Delta v_{1} / \mathrm{d} \eta$ is expected to reflect different effects, including those originating from the early time magnetic field dynamics [19,26,41] and the Coulomb interaction with charged spectators [54], as well as the transport to midrapidity via the baryon stopping mechanism [17] of the positive charge carried by the protons from the colliding

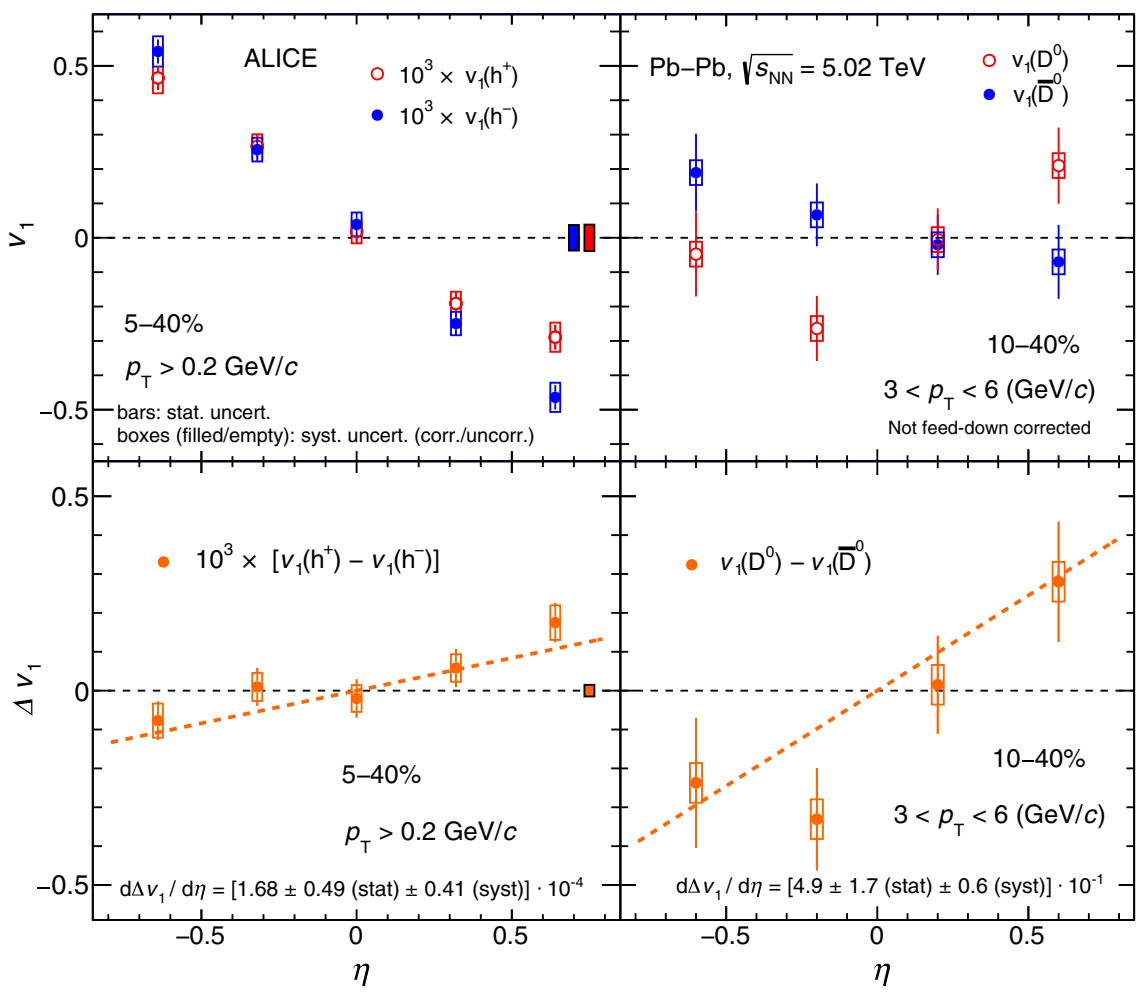

FIG. 2. Upper left: $v_{1}$ of positively (red) and negatively (blue) charged hadrons for the $5 \%-40 \%$ centrality interval. Upper right: $v_{1}$ of $\mathrm{D}^{0}$ (red) and $\overline{\mathrm{D}}^{0}$ (blue) for the $10 \%-40 \%$ centrality interval. Lower left and right: $\Delta v_{1}(h)=v_{1}\left(h^{+}\right)-v_{1}\left(h^{-}\right)$and $\Delta v_{1}(\mathrm{D})=$ $v_{1}\left(\mathrm{D}^{0}\right)-v_{1}\left(\overline{\mathrm{D}}^{0}\right)$, respectively. Dashed lines represent fits with a linear function. 
nuclei. The importance of baryon stopping for the charge dependence of unidentified hadron $v_{1}$ is supported by the observed difference, even at top RHIC energy, between proton and antiproton $v_{1}[22,55,56]$. The baryon stopping effects are expected to decrease with increasing collision energy, as supported by the observation of a smaller magnitude of $v_{1}$ [20] and of a proton to antiproton ratio closer to unity at the LHC as compared to RHIC [57]. Despite the overall decrease, the baryon stopping can contribute significantly to the proton and antiproton $v_{1}$ difference and, as such, to the charge dependence of the inclusive hadron $v_{1}$.

The charged hadron $\mathrm{d} \Delta v_{1} / \mathrm{d} \eta$ at $\sqrt{s_{\mathrm{NN}}}=5.02 \mathrm{TeV}$ is 1 order of magnitude larger and has an opposite sign with respect to calculations for charged pions at $\sqrt{s_{\mathrm{NN}}}=$ $2.76 \mathrm{TeV}$ [38] based on the analytic solution of relativistic hydrodynamics [58] with a constant electrical conductivity of the QGP. More recent calculations [54], using viscous hydrodynamic calculations [59], yield an absolute value of $\mathrm{d} \Delta v_{1} / \mathrm{d} \eta$ of similar magnitude as the one measured for charged hadrons but with opposite sign.

The $\mathrm{D}^{0}$ and $\overline{\mathrm{D}}^{0} v_{1}$ as a function of pseudorapidity is shown in the upper right panel of Fig. 2. The data suggest a positive slope for the rapidity dependence of the $v_{1}$ of $\mathrm{D}^{0}$ and a negative slope for $\overline{\mathrm{D}}^{0}$, with a significance of about $2 \sigma$ in both cases. The slopes are different from the measurements in $\mathrm{Au}-\mathrm{Au}$ collisions at $\sqrt{s_{\mathrm{NN}}}=200 \mathrm{GeV}$ [42], where a negative value is observed for both $\mathrm{D}^{0}$ and $\overline{\mathrm{D}}^{0}$. Additionally, the $v_{1}$ for $\mathrm{D}^{0}$ and $\overline{\mathrm{D}}^{0}$ mesons with $3<p_{\mathrm{T}}<$ $6 \mathrm{GeV} / c\left(\left\langle p_{\mathrm{T}}\right\rangle \approx 4.2 \mathrm{GeV} / c\right)$ in the $10 \%-40 \%$ centrality interval is about 3 orders of magnitude larger than that of charged hadrons with $p_{\mathrm{T}}>0.2 \mathrm{GeV} / c\left(\left\langle p_{\mathrm{T}}\right\rangle \approx 0.7 \mathrm{GeV} / c\right)$ in the 5\%-40\% centrality class. The different $p_{\mathrm{T}}$ intervals used for the charged hadron and $\mathrm{D}^{0}$ meson $v_{1}$ measurements are imposed by the statistical precision of the data, which simultaneously limits the yield of high $p_{\mathrm{T}}$ charged hadrons and results in low significance of the $\mathrm{D}^{0}$ and $\overline{\mathrm{D}}^{0}$ meson yield at low $p_{\mathrm{T}}$. The charged hadron $v_{1}$ at the LHC has a weak centrality dependence and changes sign around $p_{\mathrm{T}} \approx 1.5 \mathrm{GeV} / c$ [20]. The differences in centrality and transverse momentum intervals should not be responsible for the observed difference between the magnitude of the $v_{1}$ of charged hadrons and $\mathrm{D}^{0}$ and $\overline{\mathrm{D}}^{0}$ mesons. The $\mathrm{D}^{0}$ and $\overline{\mathrm{D}}^{0}$ $v_{1}$ is an order of magnitude larger than the predictions from the transport [41] and hydrodynamic $[19,26]$ model calculations. The difference between the $v_{1}$ values of $\mathrm{D}^{0}$ and $\overline{\mathrm{D}}^{0}$ mesons $\Delta v_{1}(D)$ is shown in the lower right panel of Fig. 2. The value of $\mathrm{d} \Delta v_{1} / \mathrm{d} \eta=[4.9 \pm 1.7$ (stat) \pm 0.6 (syst) $] \times 10^{-1}$ corresponds to a significance of $2.7 \sigma$ to have a positive slope. A negative value for $\mathrm{d} \Delta v_{1} / \mathrm{d} \eta$ was predicted in [41] and is observed in $\mathrm{Au}-\mathrm{Au}$ collisions at $\sqrt{s_{\mathrm{NN}}}=200 \mathrm{GeV}$ [42]. The opposite sign of the measured $\mathrm{D}^{0}$ meson and charged hadron $\Delta v_{1}$ slope with respect to model calculations might indicate a stronger effect of the
Lorentz force relative to the Coulomb one. These results demonstrate the sensitivity of the $v_{1}$ to the interplay among the effects of the rapidly decreasing magnetic field and the initial tilt of the source.

In summary, first measurements of the charge dependence of $v_{1}$ relative to the spectator plane in midcentral $\mathrm{Pb}$ $\mathrm{Pb}$ collisions at $\sqrt{s_{\mathrm{NN}}}=5.02 \mathrm{TeV}$ are presented. The $v_{1}$ and the difference $\Delta v_{1}$ between positively and negatively charged hadrons and $\mathrm{D}^{0}$ mesons are sensitive to the effects of the electromagnetic fields induced by spectator protons, baryon number transport, and the initial tilt or rotation of the particle-emitting source for noncentral collisions. An indication of a positive slope $\mathrm{d} \Delta v_{1} / \mathrm{d} \eta$ of the chargedependent $v_{1}$ at midrapidity for both charged hadrons and $\mathrm{D}^{0}$ and $\overline{\mathrm{D}}^{0}$ mesons is observed. The slope $\mathrm{d} \Delta v_{1} / \mathrm{d} \eta$ is $[1.68 \pm 0.49$ (stat) \pm 0.41 (syst) $] \times 10^{-4}$ for charged hadrons with $p_{\mathrm{T}}>0.2 \mathrm{GeV} / c$ and $[4.9 \pm 1.7$ (stat) \pm 0.6 (syst) $] \times 10^{-1}$ for $\mathrm{D}^{0}$ and $\overline{\mathrm{D}}^{0}$ mesons with $3<p_{\mathrm{T}}<6 \mathrm{GeV} / c$, with significance of $2.6 \sigma$ and $2.7 \sigma$ for having a positive value, respectively. The measured values of $v_{1}$ for $\mathrm{D}^{0}$ and $\overline{\mathrm{D}}^{0}$ mesons are about 3 orders of magnitude larger than the measured value of charged hadrons. These measurements together with those at RHIC [42] provide new insights and can constrain the theoretical modeling $[38,41]$ of electromagnetic effects. Further constraints will be set by future higher precision measurements at the LHC $[60,61]$.

ALICE Collaboration would like to thank all its engineers and technicians for their invaluable contributions to the construction of the experiment and the CERN accelerator teams for the outstanding performance of the LHC complex. ALICE Collaboration gratefully acknowledges the resources and support provided by all grid centers and the Worldwide LHC Computing Grid (WLCG) collaboration. ALICE Collaboration acknowledges the following funding agencies for their support in building and running the ALICE detector: A. I. Alikhanyan National Science Laboratory (Yerevan Physics Institute) Foundation (ANSL), State Committee of Science and World Federation of Scientists (WFS), Armenia; Austrian Academy of Sciences, Austrian Science Fund (FWF) [Grant No. M 2467-N36] and Nationalstiftung für Forschung, Technologie und Entwicklung, Austria; Ministry of Communications and High Technologies, National Nuclear Research Center, Azerbaijan; Conselho Nacional de Desenvolvimento Científico e Tecnológico (CNPq), Financiadora de Estudos e Projetos (Finep), Fundação de Amparo à Pesquisa do Estado de São Paulo (FAPESP), and Universidade Federal do Rio Grande do Sul (UFRGS), Brazil; Ministry of Education of China (MOEC), Ministry of Science and Technology of China (MSTC), and National Natural Science Foundation of China (NSFC), China; Ministry of Science and Education and Croatian Science Foundation, Croatia; Centro de Aplicaciones Tecnológicas y Desarrollo Nuclear (CEADEN), Cubaenergía, Cuba; 
Ministry of Education, Youth and Sports of the Czech Republic, Czech Republic; The Danish Council for Independent Research I Natural Sciences, the VILLUM FONDEN, and Danish National Research Foundation (DNRF), Denmark; Helsinki Institute of Physics (HIP), Finland; Commissariat à l'Energie Atomique (CEA), Institut National de Physique Nucléaire et de Physique des Particules (IN2P3), Centre National de la Recherche Scientifique (CNRS), and Région des Pays de la Loire, France; Bundesministerium für Bildung und Forschung (BMBF) and GSI Helmholtzzentrum für Schwerionenforschung GmbH, Germany; General Secretariat for Research and Technology, Ministry of Education, Research and Religions, Greece; National Research, Development and Innovation Office, Hungary; Department of Atomic Energy Government of India (DAE), Department of Science and Technology, Government of India (DST), University Grants Commission, Government of India (UGC), and Council of Scientific and Industrial Research (CSIR), India; Indonesian Institute of Science, Indonesia; Centro Fermi-Museo Storico della Fisica e Centro Studi e Ricerche Enrico Fermi and Istituto Nazionale di Fisica Nucleare (INFN), Italy; Institute for Innovative Science and Technology, Nagasaki Institute of Applied Science (IIST), Japanese Ministry of Education, Culture, Sports, Science, and Technology (MEXT) and Japan Society for the Promotion of Science (JSPS) KAKENHI, Japan; Consejo Nacional de Ciencia (CONACYT) y Tecnología through Fondo de Cooperación Internacional en Ciencia y Tecnología (FONCICYT) and Dirección General de Asuntos del Personal Academico (DGAPA), Mexico; Nederlandse Organisatie voor Wetenschappelijk Onderzoek (NWO), Netherlands; The Research Council of Norway, Norway; Commission on Science and Technology for Sustainable Development in the South (COMSATS), Pakistan; Pontificia Universidad Católica del Perú, Peru; Ministry of Science and Higher Education and National Science Centre, Poland; Korea Institute of Science and Technology Information and National Research Foundation of Korea (NRF), Republic of Korea; Ministry of Education and Scientific Research, Institute of Atomic Physics and Ministry of Research and Innovation, and Institute of Atomic Physics, Romania; Joint Institute for Nuclear Research (JINR), Ministry of Education and Science of the Russian Federation, National Research Center Kurchatov Institute, Russian Science Foundation, and Russian Foundation for Basic Research, Russia; Ministry of Education, Science, Research, and Sport of the Slovak Republic, Slovakia; National Research Foundation of South Africa, South Africa; Swedish Research Council (VR) and Knut and Alice Wallenberg Foundation (KAW), Sweden; European Organization for Nuclear Research, Switzerland; Suranaree University of Technology (SUT), National
Science and Technology Development Agency (NSDTA), and Office of the Higher Education Commission under NRU project of Thailand, Thailand; Turkish Atomic Energy Agency (TAEK), Turkey; National Academy of Sciences of Ukraine, Ukraine; Science and Technology Facilities Council (STFC), United Kingdom; National Science Foundation (NSF) and U.S. Department of Energy, Office of Nuclear Physics (DOE NP), USA.

[1] A. Bazavov et al. (HotQCD Collaboration), Chiral crossover in QCD at zero and non-zero chemical potentials, Phys. Lett. B 795, 15 (2019).

[2] A. Bazavov et al., The QCD equation of state to $\mathcal{O}\left(\mu_{B}^{6}\right)$ from lattice QCD, Phys. Rev. D 95, 054504 (2017).

[3] A. Bazavov et al. (HotQCD Collaboration), Equation of state in $(2+1)$-flavor QCD, Phys. Rev. D 90, 094503 (2014).

[4] A. Bazavov et al., The chiral and deconfinement aspects of the QCD transition, Phys. Rev. D 85, 054503 (2012).

[5] S. Borsanyi, Z. Fodor, C. Hoelbling, S. D. Katz, S. Krieg, C. Ratti, and K. K. Szabo (Wuppertal-Budapest Collaboration), Is there still any $\mathrm{T}_{\mathrm{c}}$ mystery in lattice QCD? Results with physical masses in the continuum limit III, J. High Energy Phys. 09 (2010) 073.

[6] S. Borsanyi, G. Endrodi, Z. Fodor, A. Jakovac, S. D. Katz, S. Krieg, C. Ratti, and K. K. Szabo (Wuppertal-Budapest Collaboration), The QCD equation of state with dynamical quarks, J. High Energy Phys. 11 (2010) 077.

[7] K. Aamodt et al. (ALICE Collaboration), Elliptic Flow of Charged Particles in $\mathrm{Pb}-\mathrm{Pb}$ collisions at $2.76 \mathrm{TeV}$, Phys. Rev. Lett. 105, 252302 (2010).

[8] K. Aamodt et al. (ALICE Collaboration), Higher Harmonic Anisotropic Flow Measurements of Charged Particles in $\mathrm{Pb}-\mathrm{Pb}$ Collisions at $\sqrt{s_{N N}}=2.76 \mathrm{TeV}$, Phys. Rev. Lett. 107, 032301 (2011).

[9] G. Aad et al. (ATLAS Collaboration), Measurement of the azimuthal anisotropy for charged particle production in $\sqrt{s_{N N}}=2.76 \mathrm{TeV}$ lead-lead collisions with the ATLAS detector, Phys. Rev. C 86, 014907 (2012).

[10] S. Chatrchyan et al. (CMS Collaboration), Measurement of the elliptic anisotropy of charged particles produced in $\mathrm{PbPb}$ collisions at $\sqrt{s}_{N N}=2.76 \mathrm{TeV}$, Phys. Rev. C 87, 014902 (2013).

[11] B. Abelev et al. (ALICE Collaboration), Elliptic flow of identified hadrons in $\mathrm{Pb}-\mathrm{Pb}$ collisions at $\sqrt{s_{\mathrm{NN}}}=2.76 \mathrm{TeV}$, J. High Energy Phys. 06 (2015) 190.

[12] U. Heinz and R. Snellings, Collective flow and viscosity in relativistic heavy-ion collisions, Annu. Rev. Nucl. Part. Sci. 63, 123 (2013).

[13] C. Gale, S. Jeon, and B. Schenke, Hydrodynamic modeling of heavy-ion collisions, Int. J. Mod. Phys. A 28, 1340011 (2013).

[14] H. Niemi, K. J. Eskola, and R. Paatelainen, Event-byevent fluctuations in a perturbative QCD + saturation + hydrodynamics model: Determining QCD matter shear viscosity in ultrarelativistic heavy-ion collisions, Phys. Rev. C 93, 024907 (2016). 
[15] J. E. Bernhard, J. S. Moreland, S. A. Bass, J. Liu, and U. Heinz, Applying Bayesian parameter estimation to relativistic heavy-ion collisions: Simultaneous characterization of the initial state and quark-gluon plasma medium, Phys. Rev. C 94, 024907 (2016).

[16] A. Dubla, S. Masciocchi, J. M. Pawlowski, B. Schenke, C. Shen, and J. Stachel, Towards QCD-assisted hydrodynamics for heavy-ion collision phenomenology, Nucl. Phys. A979, 251 (2018).

[17] R. J. M. Snellings, H. Sorge, S. A. Voloshin, F. Q. Wang, and N. Xu, Novel Rapidity Dependence of Directed Flow in High-Energy Heavy Ion Collisions, Phys. Rev. Lett. 84, 2803 (2000).

[18] P. Bozek and I. Wyskiel, Directed flow in ultrarelativistic heavy-ion collisions, Phys. Rev. C 81, 054902 (2010).

[19] S. Chatterjee and P. Bozek, Large Directed Flow of Open Charm Mesons Probes the Three Dimensional Distribution of Matter in Heavy Ion Collisions, Phys. Rev. Lett. 120, 192301 (2018).

[20] B. Abelev et al. (ALICE Collaboration), Directed Flow of Charged Particles at Midrapidity Relative to the Spectator Plane in Pb-Pb Collisions at $\sqrt{s_{N N}}=2.76 \mathrm{TeV}$, Phys. Rev. Lett. 111, 232302 (2013).

[21] B. I. Abelev et al. (STAR Collaboration), System-Size Independence of Directed Flow at the Relativistic HeavyIon Collider, Phys. Rev. Lett. 101, 252301 (2008).

[22] L. Adamczyk et al. (STAR Collaboration), ChargeDependent Directed Flow in $\mathrm{Cu}+\mathrm{Au}$ Collisions at $\sqrt{s_{N N}}=200 \mathrm{GeV}$, Phys. Rev. Lett. 118, 012301 (2017).

[23] A. Adil, M. Gyulassy, and T. Hirano, 3D jet tomography of the twisted color glass condensate, Phys. Rev. D 73, 074006 (2006).

[24] A. Adil and M. Gyulassy, 3D jet tomography of twisted strongly coupled quark gluon plasmas, Phys. Rev. C 72, 034907 (2005).

[25] L. P. Csernai, V. K. Magas, H. Stocker, and D. D. Strottman, Fluid dynamical prediction of changed v1-flow at LHC, Phys. Rev. C 84, 024914 (2011).

[26] S. Chatterjee and P. Bozek, Interplay of drag by hot matter and electromagnetic force on the directed flow of heavy quarks, Phys. Lett. B 798, 134955 (2019).

[27] K. Tuchin, Particle production in strong electromagnetic fields in relativistic heavy-ion collisions, Adv. High Energy Phys. 2013, 1 (2013).

[28] V. Skokov, A. Yu. Illarionov, and V. Toneev, Estimate of the magnetic field strength in heavy-ion collisions, Int. J. Mod. Phys. A 24, 5925 (2009).

[29] K. Fukushima, D. E. Kharzeev, and H. J. Warringa, The chiral magnetic effect, Phys. Rev. D 78, 074033 (2008).

[30] D. E. Kharzeev, L. D. McLerran, and H. J. Warringa, The effects of topological charge change in heavy ion collisions: 'Event by event P and CP violation', Nucl. Phys. A803, 227 (2008).

[31] K. Tuchin, Synchrotron radiation by fast fermions in heavyion collisions, Phys. Rev. C 82, 034904 (2010); Erratum, Phys. Rev. C 83, 039903 (2011).

[32] V. Voronyuk, V. D. Toneev, W. Cassing, E. L. Bratkovskaya, V. P. Konchakovski, and S. A. Voloshin, (Electro-)Magnetic field evolution in relativistic heavy-ion collisions, Phys. Rev. C 83, 054911 (2011).
[33] V. Khachatryan et al. (CMS Collaboration), Observation of charge-dependent azimuthal correlations in $p$ - $\mathrm{Pb}$ collisions and its implication for the search for the chiral magnetic effect, Phys. Rev. Lett. 118, 122301 (2017).

[34] B. Abelev et al. (ALICE Collaboration), Charge Separation Relative to the Reaction Plane in $\mathrm{Pb}-\mathrm{Pb}$ Collisions at $\sqrt{s_{N N}}=2.76 \mathrm{TeV}$, Phys. Rev. Lett. 110, 012301 (2013).

[35] B. I. Abelev et al. (STAR Collaboration), Azimuthal Charged-Particle Correlations and Possible Local Strong Parity Violation, Phys. Rev. Lett. 103, 251601 (2009).

[36] S. Schlichting and S. Pratt, Charge conservation at energies available at the BNL Relativistic Heavy Ion Collider and contributions to local parity violation observables, Phys. Rev. C 83, 014913 (2011).

[37] A. Rybicki and A. Szczurek, Spectator induced electromagnetic effect on directed flow in heavy ion collisions, Phys. Rev. C 87, 054909 (2013).

[38] U. Gursoy, D. Kharzeev, and K. Rajagopal, Magnetohydrodynamics, charged currents and directed flow in heavy ion collisions, Phys. Rev. C 89, 054905 (2014).

[39] H. T. Ding, A. Francis, O. Kaczmarek, F. Karsch, E. Laermann, and W. Soeldner, Thermal dilepton rate and electrical conductivity: An analysis of vector current correlation functions in quenched lattice QCD, Phys. Rev. D 83, 034504 (2011).

[40] A. Amato, G. Aarts, C. Allton, P. Giudice, S. Hands, and J.-I. Skullerud, Electrical conductivity of the quark-gluon plasma across the deconfinement transition, Phys. Rev. Lett. 111, 172001 (2013).

[41] S. K. Das, S. Plumari, S. Chatterjee, J. Alam, F. Scardina, and V. Greco, Directed flow of charm quarks as a witness of the initial strong magnetic field in ultra-relativistic heavy ion collisions, Phys. Lett. B 768, 260 (2017).

[42] J. Adam et al. (STAR Collaboration), First Observation of the Directed Flow of $D^{0}$ and $\overline{D^{0}}$ in $\mathrm{Au}+\mathrm{Au}$ Collisions at $\sqrt{s_{\mathrm{NN}}}=200 \mathrm{GeV}$, Phys. Rev. Lett. 123, 162301 (2019).

[43] E. Abbas et al. (ALICE Collaboration), Performance of the ALICE VZERO system, J. Instrum. 8, P10016 (2013).

[44] K. Aamodt et al. (ALICE Collaboration), The ALICE experiment at the CERN LHC, J. Instrum. 3, S08002 (2008).

[45] B. Abelev et al. (ALICE Collaboration), Performance of the ALICE Experiment at the CERN LHC, Int. J. Mod. Phys. A 29, 1430044 (2014).

[46] I. Selyuzhenkov and S. Voloshin, Effects of non-uniform acceptance in anisotropic flow measurement, Phys. Rev. C 77, 034904 (2008).

[47] M. Luzum and J.-Y. Ollitrault, Eliminating experimental bias in anisotropic-flow measurements of high-energy nuclear collisions, Phys. Rev. C 87, 044907 (2013).

[48] E. Retinskaya, M. Luzum, and J.-Y. Ollitrault, Directed flow at midrapidity in $\sqrt{s_{N N}}=2.76 \mathrm{TeV} \mathrm{Pb}+\mathrm{Pb}$ collisions, Phys. Rev. Lett. 108, 252302 (2012).

[49] K. Aamodt et al. (ALICE Collaboration), Alignment of the ALICE Inner Tracking System with cosmic-ray tracks, J. Instrum. 5, P03003 (2010).

[50] J. Alme et al., The ALICE TPC, a large 3-dimensional tracking device with fast readout for ultra-high multiplicity events, Nucl. Instrum. Methods Phys. Res., Sect. A 622, 316 (2010). 
[51] F. Carnesecchi (ALICE Collaboration), Performance of the ALICE Time-Of-Flight detector at the LHC, J. Instrum. 14, C06023 (2019).

[52] S. Acharya et al. (ALICE Collaboration), D-Meson Azimuthal Anisotropy in Midcentral $\mathrm{Pb}-\mathrm{Pb}$ Collisions at $\sqrt{\mathbf{s}_{\mathbf{N N}}}=\mathbf{5 . 0 2}$ TeV, Phys. Rev. Lett. 120, 102301 (2018).

[53] S. Acharya et al. (ALICE Collaboration), Measurement of $\mathrm{D}^{0}, D^{+}, \mathrm{D}^{*+}$ and $\mathrm{D}_{s}^{+}$production in $\mathrm{Pb}-\mathrm{Pb}$ collisions at $\sqrt{\mathrm{s}_{\mathrm{NN}}}=5.02 \mathrm{TeV}$, J. High Energy Phys. 10 (2018) 174.

[54] U. Gursoy, D. Kharzeev, E. Marcus, K. Rajagopal, and C. Shen, Charge-dependent flow induced by magnetic and electric fields in heavy ion collisions, Phys. Rev. C 98, 055201 (2018).

[55] L. Adamczyk et al. (STAR Collaboration), Directed Flow of Identified Particles in $\mathrm{Au}+\mathrm{Au}$ Collisions at $\sqrt{s_{N N}}=200 \mathrm{GeV}$ at RHIC, Phys. Rev. Lett. 108, 202301 (2012).

[56] L. Adamczyk et al. (STAR Collaboration), Beam-Energy Dependence of the Directed Flow of Protons, Antiprotons, and Pions in $\mathrm{Au}+\mathrm{Au}$ Collisions, Phys. Rev. Lett. 112, 162301 (2014).

[57] B. Abelev et al. (ALICE Collaboration), Centrality dependence of $\pi, \mathrm{K}, \mathrm{p}$ production in $\mathrm{Pb}-\mathrm{Pb}$ collisions at $\sqrt{s_{N N}}=2.76 \mathrm{TeV}$, Phys. Rev. C 88, 044910 (2013).

[58] S. S. Gubser, Symmetry constraints on generalizations of Bjorken flow, Phys. Rev. D 82, 085027 (2010).

[59] C. Shen, Z. Qiu, H. Song, J. Bernhard, S. Bass, and U. Heinz, The iEBE-VISHNU code package for relativistic heavy-ion collisions, Comput. Phys. Commun. 199, 61 (2016).

[60] Z. Citron et al., Future physics opportunities for highdensity QCD at the LHC with heavy-ion and proton beams, in HL/HE-LHC Workshop: Workshop on the Physics of HLLHC, and Perspectives at HE-LHC Geneva, Switzerland, 2018 (2018).

[61] S. Acharya et al. (ALICE Collaboration), ALICE upgrade physics performance studies for 2018 Report on HL/HELHC physics, Report No. ALICE-PUBLIC-2019-001 (2019), https://cds.cern.ch/record/2661798.

S. Acharya, ${ }^{141}$ D. Adamová, ${ }^{94}$ A. Adler, ${ }^{74}$ J. Adolfsson, ${ }^{80}$ M. M. Aggarwal,${ }^{99}$ G. Aglieri Rinella, ${ }^{33}$ M. Agnello ${ }^{30}$ N. Agrawal,${ }^{10,53}$ Z. Ahammed, ${ }^{141}$ S. Ahmad, ${ }^{16}$ S. U. Ahn, ${ }^{76}$ A. Akindinov, ${ }^{91}$ M. Al-Turany, ${ }^{106}$ S. N. Alam, ${ }^{141}$ D. S. D. Albuquerque, ${ }^{122}$ D. Aleksandrov, ${ }^{87}$ B. Alessandro, ${ }^{58}$ H. M. Alfanda, ${ }^{6}$ R. Alfaro Molina, ${ }^{71}$ B. Ali, ${ }^{16}$ Y. Ali, ${ }^{14}$ A. Alici, ${ }^{10,26 a, 26 b, 53}$ A. Alkin, ${ }^{2}$ J. Alme, ${ }^{21}$ T. Alt, ${ }^{68}$ L. Altenkamper, ${ }^{21}$ I. Altsybeev, ${ }^{112}$ M. N. Anaam, ${ }^{6}$ C. Andrei, ${ }^{47}$ D. Andreou, ${ }^{33}$ H. A. Andrews, ${ }^{110}$ A. Andronic, ${ }^{144}$ M. Angeletti, ${ }^{33}$ V. Anguelov, ${ }^{103}$ C. Anson, ${ }^{15}$ T. Antičić, ${ }^{107}$ F. Antinori ${ }^{56}$ P. Antonioli, ${ }^{53}$ R. Anwar, ${ }^{125}$ N. Apadula,${ }^{79}$ L. Aphecetche, ${ }^{114}$ H. Appelshäuser, ${ }^{68}$ S. Arcelli, ${ }^{26 a, 26 b}$ R. Arnaldi,${ }^{58}$ M. Arratia, ${ }^{79}$ I. C. Arsene, ${ }^{20}$ M. Arslandok, ${ }^{103}$ A. Augustinus, ${ }^{33}$ R. Averbeck, ${ }^{106}$ S. Aziz,${ }^{61}$ M. D. Azmi, ${ }^{16}$ A. Badalà, ${ }^{55}$ Y. W. Baek, ${ }^{40}$ S. Bagnasco, ${ }^{58}$ X. Bai, ${ }^{106}$ R. Bailhache,${ }^{68}$ R. Bala, ${ }^{100}$ A. Baldisseri, ${ }^{137}$ M. Ball, ${ }^{42}$ S. Balouza,${ }^{104}$ R. Barbera, ${ }^{27 a, 27 b}$ L. Barioglio, ${ }^{25 a, 25 b}$ G. G. Barnaföldi, ${ }^{145}$ L. S. Barnby, ${ }^{93}$ V. Barret, ${ }^{134}$ P. Bartalini, ${ }^{6}$ K. Barth, ${ }^{33}$ E. Bartsch,${ }^{68}$ F. Baruffaldi, ${ }^{28,28 b}$ N. Bastid, ${ }^{134}$ S. Basu, ${ }^{143}$ G. Batigne, ${ }^{114}$ B. Batyunya,${ }^{75}$ D. Bauri, ${ }^{48}$ J. L. Bazo Alba, ${ }^{111}$ I. G. Bearden, ${ }^{88}$ C. Bedda ${ }^{63}$ N. K. Behera, ${ }^{60}$ I. Belikov ${ }^{136}$ A. D. C. Bell Hechavarria,${ }^{144}$ F. Bellini, ${ }^{33}$ R. Bellwied, ${ }^{125}$ V. Belyaev, ${ }^{92}$ G. Bencedi, ${ }^{145}$ S. Beole,${ }^{25 a, 25 b}$ A. Bercuci,${ }^{47}$ Y. Berdnikov, ${ }^{97}$ D. Berenyi,${ }^{145}$ R. A. Bertens, ${ }^{130}$ D. Berzano,${ }^{58}$ M. G. Besoiu, ${ }^{67}$ L. Betev ${ }^{33}$ A. Bhasin, ${ }^{100}$ I. R. Bhat, ${ }^{100}$ M. A. Bhat,${ }^{3 a, 3 b}$ H. Bhatt, ${ }^{48}$ B. Bhattacharjee, ${ }^{41}$ A. Bianchi, ${ }^{25 a, 25 b}$ L. Bianchi, ${ }^{25 a, 25 b}$ N. Bianchi, ${ }^{51}$ J. Bielč́́k, ${ }^{36}$ J. Bielč́́ková, ${ }^{94}$ A. Bilandzic, ${ }^{104,117}$ G. Biro, ${ }^{145}$ R. Biswas, ${ }^{3 a, 3 b}$ S. Biswas, ${ }^{3 a, 3 b}$ J. T. Blair, ${ }^{119}$ D. Blau, ${ }^{87}$ C. Blume ${ }^{68}$ G. Boca, ${ }^{139}$ F. Bock, ${ }^{33,95}$ A. Bogdanov, ${ }^{92}$ S. Boi, ${ }^{23 a, 23 b}$ L. Boldizsár, ${ }^{145}$ A. Bolozdynya, ${ }^{92}$ M. Bombara, ${ }^{37}$ G. Bonomi, ${ }^{140}$ H. Borel,,${ }^{137}$ A. Borissov, ${ }^{92,144}$ H. Bossi, ${ }^{146}$ E. Botta, ${ }^{25 a, 25 b}$ L. Bratrud, ${ }^{68}$

P. Braun-Munzinger, ${ }^{106}$ M. Bregant, ${ }^{121}$ M. Broz, ${ }^{36}$ E. J. Brucken, ${ }^{43}$ E. Bruna,${ }^{58}$ G. E. Bruno, ${ }^{105}$ M. D. Buckland, ${ }^{127}$ D. Budnikov, ${ }^{108}$ H. Buesching, ${ }^{68}$ S. Bufalino, ${ }^{30}$ O. Bugnon, ${ }^{114}$ P. Buhler,${ }^{113}$ P. Buncic, ${ }^{33}$ Z. Buthelezi, ${ }^{72,131}$ J. B. Butt, ${ }^{14}$ J. T. Buxton, ${ }^{96}$ S. A. Bysiak, ${ }^{118}$ D. Caffarri, ${ }^{89}$ A. Caliva, ${ }^{106}$ E. Calvo Villar, ${ }^{111}$ R. S. Camacho, ${ }^{44}$ P. Camerini, ${ }^{24 a, 24 b}$ A. A. Capon, ${ }^{113}$ F. Carnesecchi, ${ }^{10,26 a, 26 b}$ R. Caron,${ }^{137}$ J. Castillo Castellanos,${ }^{137}$ A. J. Castro, ${ }^{130}$ E. A. R. Casula, ${ }^{54}$ F. Catalano, ${ }^{30}$ C. Ceballos Sanchez, ${ }^{52}$ P. Chakraborty, ${ }^{48}$ S. Chandra, ${ }^{141}$ W. Chang, ${ }^{6}$ S. Chapeland, ${ }^{33}$ M. Chartier, ${ }^{127}$ S. Chattopadhyay, ${ }^{141}$ S. Chattopadhyay, ${ }^{109}$ A. Chauvin, ${ }^{23 a, 23 b}$ C. Cheshkov, ${ }^{135}$ B. Cheynis, ${ }^{135}$ V. Chibante Barroso, ${ }^{33}$ D. D. Chinellato, ${ }^{122}$ S. Cho ${ }^{60}$ P. Chochula, ${ }^{33}$ T. Chowdhury,${ }^{134}$ P. Christakoglou, ${ }^{89}$ C. H. Christensen,${ }^{88}$ P. Christiansen,${ }^{80}$ T. Chujo, ${ }^{133}$ C. Cicalo ${ }^{54}$ L. Cifarelli, ${ }^{10,26 a, 26 b}$ F. Cindolo, ${ }^{53}$ J. Cleymans, ${ }^{124}$ F. Colamaria,${ }^{52}$ D. Colella, ${ }^{52}$ A. Collu, ${ }^{79}$ M. Colocci, ${ }^{26 a, 26 b}$ M. Concas, ${ }^{58, b}$ G. Conesa Balbastre, ${ }^{78}$ Z. Conesa del Valle, ${ }^{61}$ G. Contin, ${ }^{24 a, 24 b, 127}$ J. G. Contreras, ${ }^{36}$ T. M. Cormier, ${ }^{95}$ Y. Corrales Morales, ${ }^{25 a, 25 b}$ P. Cortese, ${ }^{31}$ M. R. Cosentino, ${ }^{123}$ F. Costa, ${ }^{33}$ S. Costanza, ${ }^{139}$ P. Crochet, ${ }^{134}$ E. Cuautle,${ }^{69}$ P. Cui, ${ }^{6}$ L. Cunqueiro, ${ }^{95}$ D. Dabrowski, ${ }^{142}$ T. Dahms, ${ }^{104,117}$ A. Dainese, ${ }^{56}$ F. P. A. Damas, ${ }^{114,137}$ M. C. Danisch, ${ }^{103}$ A. Danu, ${ }^{67}$ D. Das, ${ }^{109}$ I. Das, ${ }^{109}$ P. Das, ${ }^{85}$ P. Das, ${ }^{3 a, 3 b}$ S. Das, ${ }^{3 a, 3 b}$ A. Dash,${ }^{85}$ S. Dash,${ }^{48}$ S. De,${ }^{85}$ A. De Caro, ${ }^{29 a, 29 b}$ G. de Cataldo, ${ }^{52}$ J. de Cuveland, ${ }^{38}$ A. De Falco, ${ }^{23 a, 23 b}$ D. De Gruttola, ${ }^{10}$ N. De Marco ${ }^{58}$

S. De Pasquale, ${ }^{29 a, 29 b}$ S. Deb, ${ }^{49}$ B. Debjani, ${ }^{3 \mathrm{a}, 3 \mathrm{~b}}$ H. F. Degenhardt, ${ }^{121}$ K. R. Deja, ${ }^{142}$ A. Deloff, ${ }^{84}$ S. Delsanto, ${ }^{25 a, 25 b, 131}$ 
D. Devetak, ${ }^{106}$ P. Dhankher, ${ }^{48}$ D. Di Bari, ${ }^{32 a, 32 b}$ A. Di Mauro, ${ }^{33}$ R. A. Diaz, ${ }^{8}$ T. Dietel, ${ }^{124}$ P. Dillenseger, ${ }^{68}$ Y. Ding, ${ }^{6}$ R. Divià, ${ }^{33}$ D. U. Dixit, ${ }^{19} \varnothing$. Djuvsland, ${ }^{21}$ U. Dmitrieva, ${ }^{62}$ A. Dobrin,,${ }^{33,67}$ B. Dönigus, ${ }^{68}$ O. Dordic, ${ }^{20}$ A. K. Dubey, ${ }^{141}$ A. Dubla, ${ }^{106,63}$ S. Dudi, ${ }^{99}$ M. Dukhishyam, ${ }^{85}$ P. Dupieux, ${ }^{134}$ R. J. Ehlers, ${ }^{146}$ V. N. Eikeland, ${ }^{21}$ D. Elia,${ }^{52}$ H. Engel,${ }^{74}$ E. Epple,${ }^{146}$ B. Erazmus, ${ }^{114}$ F. Erhardt, ${ }^{98}$ A. Erokhin, ${ }^{112}$ M. R. Ersdal, ${ }^{21}$ B. Espagnon, ${ }^{61}$ S. Esumi, ${ }^{133}$ G. Eulisse, ${ }^{33}$ D. Evans,${ }^{110}$ S. Evdokimov,${ }^{90}$ L. Fabbietti, ${ }^{104,117}$ M. Faggin, ${ }^{28 a, 28 b}$ J. Faivre, ${ }^{78}$ F. Fan, ${ }^{6}$ A. Fantoni, ${ }^{51}$ M. Fasel,,${ }^{95}$ P. Fecchio, ${ }^{30}$ A. Feliciello, ${ }^{58}$ G. Feofilov, ${ }^{12}$ A. Fernández Téllez, ${ }^{44}$ A. Ferrero, ${ }^{137}$ A. Ferretti, ${ }^{25 a, 25 b}$ A. Festanti ${ }^{33}$ V. J. G. Feuillard, ${ }^{103}$ J. Figiel, ${ }^{118}$ S. Filchagin, ${ }^{108}$ D. Finogeev, ${ }^{62}$ F. M. Fionda, ${ }^{21}$ G. Fiorenza, ${ }^{52}$ F. Flor, ${ }^{125}$ S. Foertsch, ${ }^{72}$ P. Foka, ${ }^{106}$ S. Fokin,${ }^{87}$ E. Fragiacomo, ${ }^{59}$ U. Frankenfeld, ${ }^{106}$ U. Fuchs,${ }^{33}$ C. Furget ${ }^{78}$ A. Furs, ${ }^{62}$ M. Fusco Girard, ${ }^{29 a, 29 b}$ J. J. Gaardhøje ${ }^{88}$ M. Gagliardi, ${ }^{25 a, 25 b}$ A. M. Gago, ${ }^{111}$ A. Gal, ${ }^{136}$ C. D. Galvan, ${ }^{120}$ P. Ganoti ${ }^{83}$ C. Garabatos, ${ }^{106}$ E. Garcia-Solis, ${ }^{11}$ K. Garg, ${ }^{27 a, 27 b}$ C. Gargiulo, ${ }^{33}$ A. Garibli, ${ }^{86}$ K. Garner, ${ }^{144}$ P. Gasik, ${ }^{104,117}$ E. F. Gauger, ${ }^{119}$ M. B. Gay Ducati, ${ }^{70}$ M. Germain, ${ }^{114}$ J. Ghosh, ${ }^{109}$ P. Ghosh, ${ }^{141}$ S. K. Ghosh, ${ }^{3 a, 3 b}$ P. Gianotti, ${ }^{51}$ P. Giubellino, ${ }^{58,106}$ P. Giubilato, ${ }^{28 a, 28 b}$ P. Glässel, ${ }^{103}$ D. M. Goméz Coral, ${ }^{71}$ A. Gomez Ramirez ${ }^{74}$ V. Gonzalez, ${ }^{106}$ P. González-Zamora, ${ }^{44}$ S. Gorbunov, ${ }^{38}$ L. Görlich,${ }^{18}$ S. Gotovac, ${ }^{34}$ V. Grabski, ${ }^{71}$ L. K. Graczykowski, ${ }^{142}$ K. L. Graham, ${ }^{110}$ L. Greiner, ${ }^{79}$ A. Grelli, ${ }^{63}$ C. Grigoras, ${ }^{33}$ V. Grigoriev, ${ }^{92}$ A. Grigoryan, ${ }^{1}$ S. Grigoryan, ${ }^{75}$ O. S. Groettvik, ${ }^{21}$ F. Grosa, ${ }^{30}$ J. F. Grosse-Oetringhaus, ${ }^{33}$ R. Grosso, ${ }^{106}$ R. Guernane, ${ }^{78}$ M. Guittiere, ${ }^{114}$ K. Gulbrandsen, ${ }^{88}$ T. Gunji, ${ }^{132}$ A. Gupta, ${ }^{100}$ R. Gupta, ${ }^{100}$ I. B. Guzman, ${ }^{44}$ R. Haake, ${ }^{146}$ M. K. Habib ${ }^{106}$ C. Hadjidakis, ${ }^{61}$ H. Hamagaki, ${ }^{81}$ G. Hamar, ${ }^{145}$ M. Hamid, ${ }^{6}$ R. Hannigan, ${ }^{119}$ M. R. Haque, ${ }^{63,85}$ A. Harlenderova, ${ }^{106}$ J. W. Harris, ${ }^{146}$ A. Harton, ${ }^{11}$ J. A. Hasenbichler, ${ }^{33}$ H. Hassan, ${ }^{95}$ D. Hatzifotiadou, ${ }^{10,53}$ P. Hauer, ${ }^{42}$ S. Hayashi, ${ }^{132}$ S. T. Heckel, ${ }^{68,104}$ E. Hellbär, ${ }^{68}$ H. Helstrup, ${ }^{35}$ A. Herghelegiu, ${ }^{47}$ T. Herman, ${ }^{36}$ E. G. Hernandez, ${ }^{44}$ G. Herrera Corral, ${ }^{9}$ F. Herrmann, ${ }^{144}$ K. F. Hetland, ${ }^{35}$ T. E. Hilden, ${ }^{43}$ H. Hillemanns, ${ }^{33}$ C. Hills, ${ }^{127}$ B. Hippolyte, ${ }^{136}$ B. Hohlweger, ${ }^{104}$ D. Horak,${ }^{36}$ A. Hornung, ${ }^{68}$ S. Hornung, ${ }^{106}$ R. Hosokawa, ${ }^{15,133}$ P. Hristov,${ }^{33}$ C. Huang, ${ }^{61}$ C. Hughes,${ }^{130}$ P. Huhn, ${ }^{68}$ T. J. Humanic,${ }^{96}$ H. Hushnud, ${ }^{109}$ L. A. Husova, ${ }^{144}$ N. Hussain, ${ }^{41}$ S. A. Hussain, ${ }^{14}$ D. Hutter, ${ }^{38}$ J. P. Iddon, ${ }^{33,127}$ R. Ilkaev, ${ }^{108}$ M. Inaba, ${ }^{133}$ G. M. Innocenti, ${ }^{33}$ M. Ippolitov ${ }^{87}$ A. Isakov, ${ }^{94}$ M. S. Islam, ${ }^{109}$ M. Ivanov, ${ }^{106}$ V. Ivanov, ${ }^{97}$ V. Izucheev,${ }^{90}$ B. Jacak,${ }^{79}$ N. Jacazio,${ }^{53}$ P. M. Jacobs,${ }^{79}$ S. Jadlovska, ${ }^{116}$ J. Jadlovsky, ${ }^{116}$ S. Jaelani, ${ }^{63}$ C. Jahnke, ${ }^{121}$ M. J. Jakubowska, ${ }^{142}$ M. A. Janik,${ }^{142}$ T. Janson, ${ }^{74}$ M. Jercic, ${ }^{98}$ O. Jevons, ${ }^{110}$ M. Jin, ${ }^{125}$ F. Jonas, ${ }^{95,144}$ P. G. Jones, ${ }^{110}$ J. Jung, ${ }^{68}$ M. Jung, ${ }^{68}$ A. Jusko, ${ }^{110}$ P. Kalinak, ${ }^{64}$ A. Kalweit,${ }^{33}$

V. Kaplin, ${ }^{92}$ S. Kar, ${ }^{6}$ A. Karasu Uysal, ${ }^{77}$ O. Karavichev, ${ }^{62}$ T. Karavicheva, ${ }^{62}$ P. Karczmarczyk,${ }^{33}$ E. Karpechev, ${ }^{62}$ A. Kazantsev, ${ }^{87}$ U. Kebschull, ${ }^{74}$ R. Keidel, ${ }^{46}$ M. Keil,${ }^{33}$ B. Ketzer, ${ }^{42}$ Z. Khabanova, ${ }^{89}$ A. M. Khan, ${ }^{6}$ S. Khan, ${ }^{16}$ S. A. Khan, ${ }^{141}$ A. Khanzadeev, ${ }^{97}$ Y. Kharlov, ${ }^{90}$ A. Khatun, ${ }^{16}$ A. Khuntia, ${ }^{118}$ B. Kileng, ${ }^{35}$ B. Kim, ${ }^{60}$ B. Kim, ${ }^{133}$ D. Kim, ${ }^{147}$ D. J. Kim, ${ }^{126}$ E. J. Kim, ${ }^{73}$ H. Kim, ${ }^{17,147}$ J. Kim, ${ }^{147}$ J. S. Kim,${ }^{40}$ J. Kim,${ }^{103}$ J. Kim, ${ }^{147}$ J. Kim,${ }^{73}$ M. Kim, ${ }^{103}$ S. Kim, ${ }^{18}$ T. Kim, ${ }^{147}$ T. Kim, ${ }^{147}$ S. Kirsch, ${ }^{38,68}$ I. Kisel, ${ }^{38}$ S. Kiselev, ${ }^{91}$ A. Kisiel, ${ }^{142}$ J. L. Klay, ${ }^{5}$ C. Klein ${ }^{68}$ J. Klein, ${ }^{58}$ S. Klein, ${ }^{79}$ C. Klein-Bösing, ${ }^{144}$ M. Kleiner, ${ }^{68}$ A. Kluge ${ }^{33}$ M. L. Knichel, ${ }^{33}$ A. G. Knospe, ${ }^{125}$ C. Kobdaj, ${ }^{115}$ M. K. Köhler, ${ }^{103}$ T. Kollegger, ${ }^{106}$ A. Kondratyev, ${ }^{75}$ N. Kondratyeva, ${ }^{92}$ E. Kondratyuk, ${ }^{90}$ J. Konig, ${ }^{68}$ P. J. Konopka, ${ }^{33}$ L. Koska, ${ }^{116}$ O. Kovalenko, ${ }^{84}$ V. Kovalenko, ${ }^{112}$ M. Kowalski, ${ }^{118}$ I. Králik, ${ }^{64}$ A. Kravčáková, ${ }^{37}$ L. Kreis,${ }^{106}$ M. Krivda, ${ }^{64,110}$ F. Krizek, ${ }^{94}$

K. Krizkova Gajdosova, ${ }^{36}$ M. Krüger,${ }^{68}$ E. Kryshen, ${ }^{97}$ M. Krzewicki, ${ }^{38}$ A. M. Kubera, ${ }^{96}$ V. Kučera, ${ }^{60}$ C. Kuhn, ${ }^{136}$ P. G. Kuijer, ${ }^{89}$ L. Kumar, ${ }^{99}$ S. Kumar, ${ }^{48}$ S. Kundu, ${ }^{85}$ P. Kurashvili, ${ }^{84}$ A. Kurepin, ${ }^{62}$ A. B. Kurepin, ${ }^{62}$ A. Kuryakin,${ }^{108}$ S. Kushpil,${ }^{94}$ J. Kvapil, ${ }^{110}$ M. J. Kweon, ${ }^{60}$ J. Y. Kwon, ${ }^{60}$ Y. Kwon, ${ }^{147}$ S. L. La Pointe, ${ }^{38}$ P. La Rocca, ${ }^{27 a, 27 b}$ Y. S. Lai, ${ }^{79}$ R. Langoy, ${ }^{129}$ K. Lapidus, ${ }^{33}$ A. Lardeux,${ }^{20}$ P. Larionov, ${ }^{51}$ E. Laudi, ${ }^{33}$ R. Lavicka, ${ }^{36}$ T. Lazareva, ${ }^{112}$ R. Lea, ${ }^{24 a, 24 b}$ L. Leardini, ${ }^{103}$ J. Lee, ${ }^{133}$ S. Lee, ${ }^{147}$ F. Lehas, ${ }^{89}$ S. Lehner, ${ }^{113}$ J. Lehrbach, ${ }^{38}$ R. C. Lemmon, ${ }^{93}$ I. León Monzón, ${ }^{120}$ E. D. Lesser, ${ }^{19}$ M. Lettrich, ${ }^{33}$ P. Lévai, ${ }^{145}$ X. Li, ${ }^{12}$ X. L. Li,${ }^{6}$ J. Lien, ${ }^{129}$ R. Lietava, ${ }^{110}$ B. Lim, ${ }^{17}$ V. Lindenstruth, ${ }^{38}$ S. W. Lindsay, ${ }^{127}$ C. Lippmann, ${ }^{106}$ M. A. Lisa, ${ }^{96}$ V. Litichevskyi, ${ }^{43}$ A. Liu, ${ }^{19}$ S. Liu ${ }^{96}$ W. J. Llope,${ }^{143}$ I. M. Lofnes, ${ }^{21}$ V. Loginov, ${ }^{92}$ C. Loizides, ${ }^{95}$ P. Loncar,${ }^{34}$ X. Lopez,${ }^{134}$ E. López Torres, ${ }^{8}$ J. R. Luhder, ${ }^{144}$ M. Lunardon, ${ }^{28 a, 28 b}$ G. Luparello, ${ }^{59}$ Y. Ma, ${ }^{39}$ A. Maevskaya, ${ }^{62}$ M. Mager ${ }^{33}$ S. M. Mahmood, ${ }^{20}$ T. Mahmoud, ${ }^{42}$ A. Maire, ${ }^{136}$ R. D. Majka, ${ }^{146}$ M. Malaev, ${ }^{97}$ Q. W. Malik, ${ }^{20}$ L. Malinina,${ }^{75, \mathrm{c}}$ D. Mal'Kevich, ${ }^{91}$ P. Malzacher, ${ }^{106}$ G. Mandaglio, ${ }^{55}$ V. Manko ${ }^{87}$ F. Manso, ${ }^{134}$ V. Manzari, ${ }^{52}$ Y. Mao, ${ }^{6}$ M. Marchisone, ${ }^{135}$ J. Mareš ${ }^{66}$ G. V. Margagliotti, ${ }^{24 a, 24 b}$ A. Margotti, ${ }^{53}$ J. Margutti, ${ }^{63}$ A. Marín, ${ }^{106}$ C. Markert, ${ }^{119}$ M. Marquard, ${ }^{68}$ N. A. Martin, ${ }^{103}$ P. Martinengo, ${ }^{33}$ J. L. Martinez, ${ }^{125}$ M. I. Martínez, ${ }^{44}$ G. Martínez García, ${ }^{114}$ M. Martinez Pedreira, ${ }^{33}$ S. Masciocchi, ${ }^{106}$ M. Masera, ${ }^{25 a, 25 b}$ A. Masoni, ${ }^{54}$ L. Massacrier,${ }^{61}$ E. Masson, ${ }^{114}$ A. Mastroserio, ${ }^{52,138}$ A. M. Mathis, ${ }^{104,117}$ O. Matonoha, ${ }^{80}$ P. F. T. Matuoka, ${ }^{121}$ A. Matyja, ${ }^{118}$ C. Mayer, ${ }^{118}$ M. Mazzilli, ${ }^{52}$ M. A. Mazzoni, ${ }^{57}$ A. F. Mechler, ${ }^{68}$ F. Meddi, ${ }^{22 a, 22 b}$ Y. Melikyan, ${ }^{62,92}$ A. Menchaca-Rocha, ${ }^{71}$ C. Mengke, ${ }^{6}$

E. Meninno, ${ }^{29 a, 29 b, 113}$ M. Meres, ${ }^{13}$ S. Mhlanga, ${ }^{124}$ Y. Miake, ${ }^{133}$ L. Micheletti, ${ }^{25 a, 25 b}$ D. L. Mihaylov, ${ }^{104}$ K. Mikhaylov, ${ }^{75,91}$ A. Mischke, ${ }^{63, a}$ A. N. Mishra, ${ }^{69}$ D. Miśkowiec, ${ }^{106}$ A. Modak, ${ }^{3 a, 3 b}$ N. Mohammadi, ${ }^{33}$ A. P. Mohanty, ${ }^{63}$ B. Mohanty, ${ }^{85}$ 
M. Mohisin Khan, ${ }^{16, d}$ C. Mordasini, ${ }^{104}$ D. A. Moreira De Godoy, ${ }^{144}$ L. A. P. Moreno, ${ }^{44}$ I. Morozov, ${ }^{62}$ A. Morsch, ${ }^{33}$ T. Mrnjavac, ${ }^{33}$ V. Muccifora, ${ }^{51}$ E. Mudnic, ${ }^{34}$ D. Mühlheim, ${ }^{144}$ S. Muhuri, ${ }^{141}$ J. D. Mulligan,${ }^{79}$ M. G. Munhoz, ${ }^{121}$ R. H. Munzer, ${ }^{68}$ H. Murakami, ${ }^{132}$ S. Murray, ${ }^{124}$ L. Musa,${ }^{33}$ J. Musinsky,${ }^{64}$ C. J. Myers, ${ }^{125}$ J. W. Myrcha, ${ }^{142}$ B. Naik, ${ }^{48}$ R. Nair, ${ }^{84}$ B. K. Nandi, ${ }^{48}$ R. Nania,${ }^{10,53}$ E. Nappi, ${ }^{52}$ M. U. Naru, ${ }^{14}$ A. F. Nassirpour, ${ }^{80}$ C. Nattrass, ${ }^{130}$ R. Nayak, ${ }^{48}$ T. K. Nayak, ${ }^{85}$ S. Nazarenko, ${ }^{108}$ A. Neagu, ${ }^{20}$ R. A. Negrao De Oliveira, ${ }^{68}$ L. Nellen,${ }^{69}$ S. V. Nesbo, ${ }^{35}$ G. Neskovic, ${ }^{38}$ D. Nesterov, ${ }^{112}$ L. T. Neumann, ${ }^{142}$ B. S. Nielsen, ${ }^{88}$ S. Nikolaev, ${ }^{87}$ S. Nikulin,${ }^{87}$ V. Nikulin, ${ }^{97}$ F. Noferini, ${ }^{10,53}$ P. Nomokonov, ${ }^{75}$ J. Norman,${ }^{78,127}$ N. Novitzky, ${ }^{133}$ P. Nowakowski, ${ }^{142}$ A. Nyanin,${ }^{87}$ J. Nystrand, ${ }^{21}$ M. Ogino, ${ }^{81}$ A. Ohlson, ${ }^{80,103}$ J. Oleniacz, ${ }^{142}$ A. C. Oliveira Da Silva, ${ }^{121,130}$ M. H. Oliver,${ }^{146}$ C. Oppedisano, ${ }^{58}$ R. Orava, ${ }^{43}$ A. Ortiz Velasquez, ${ }^{69}$ A. Oskarsson, ${ }^{80}$ J. Otwinowski, ${ }^{118}$ K. Oyama, ${ }^{81}$ Y. Pachmayer, ${ }^{103}$ V. Pacik, ${ }^{88}$ D. Pagano, ${ }^{140}$ G. Paić,${ }^{69}$ J. Pan, ${ }^{143}$ A. K. Pandey, ${ }^{48}$ S. Panebianco, ${ }^{137}$ P. Pareek, ${ }^{49,141}$ J. Park, ${ }^{60}$ J. E. Parkkila, ${ }^{126}$ S. Parmar, ${ }^{99}$ S. P. Pathak, ${ }^{125}$ R. N. Patra, ${ }^{141}$ B. Paul, ${ }^{23 a, 23 b, 58}$ H. Pei, ${ }^{6}$ T. Peitzmann, ${ }^{63}$ X. Peng, ${ }^{6}$ L. G. Pereira ${ }^{70}$ H. Pereira Da Costa, ${ }^{137}$ D. Peresunko, ${ }^{87}$ G. M. Perez,${ }^{8}$ E. Perez Lezama, ${ }^{68}$ V. Peskov ${ }^{68}$ Y. Pestov ${ }^{4}$ V. Petráček, ${ }^{36}$ M. Petrovici, ${ }^{47}$ R. P. Pezzi, ${ }^{70}$ S. Piano,${ }^{59}$ M. Pikna, ${ }^{13}$ P. Pillot, ${ }^{114}$ O. Pinazza, ${ }^{33,53}$ L. Pinsky, ${ }^{125}$ C. Pinto, ${ }^{27 a, 27 b}$ S. Pisano, ${ }^{10,51}$ D. Pistone,${ }^{55}$ M. Płoskoń, ${ }^{79}$ M. Planinic, ${ }^{98}$ F. Pliquett, ${ }^{68}$ J. Pluta, ${ }^{142}$ S. Pochybova, ${ }^{145, a}$ M. G. Poghosyan, ${ }^{95}$ B. Polichtchouk, ${ }^{90}$ N. Poljak,${ }^{98}$ A. Pop, ${ }^{47}$ H. Poppenborg, ${ }^{144}$ S. Porteboeuf-Houssais, ${ }^{134}$ V. Pozdniakov, ${ }^{75}$ S. K. Prasad,${ }^{3 a, 3 b}$ R. Preghenella, ${ }^{53}$ F. Prino, ${ }^{58}$ C. A. Pruneau, ${ }^{143}$ I. Pshenichnov, ${ }^{62}$ M. Puccio, ${ }^{25 a, 25 b, 33}$ J. Putschke, ${ }^{143}$ R. E. Quishpe, ${ }^{125}$ S. Ragoni ${ }^{110}$ S. Raha ${ }^{3 a, 3 b}$ S. Rajput, ${ }^{100}$ J. Rak, ${ }^{126}$ A. Rakotozafindrabe, ${ }^{137}$ L. Ramello, ${ }^{31}$ F. Rami, ${ }^{136}$ R. Raniwala, ${ }^{101}$ S. Raniwala, ${ }^{101}$ S. S. Räsänen, ${ }^{43}$ R. Rath, ${ }^{49}$ V. Ratza, ${ }^{42}$ I. Ravasenga, ${ }^{30,89}$ K. F. Read, ${ }^{95,130}$ K. Redlich, ${ }^{84, e}$ A. Rehman, ${ }^{21}$ P. Reichelt, ${ }^{68}$ F. Reidt, ${ }^{33}$ X. Ren, ${ }^{6}$ R. Renfordt, ${ }^{68}$ Z. Rescakova, ${ }^{37}$ J.-P. Revol, ${ }^{10}$ K. Reygers, ${ }^{103}$ V. Riabov, ${ }^{97}$ T. Richert, ${ }^{80,88}$ M. Richter, ${ }^{20}$ P. Riedler, ${ }^{33}$ W. Riegler, ${ }^{33}$ F. Riggi, ${ }^{27 a, 27 b}$ C. Ristea, ${ }^{67}$ S. P. Rode, ${ }^{49}$ M. Rodríguez Cahuantzi, ${ }^{44}$ K. Røed, ${ }^{20}$ R. Rogalev, ${ }^{90}$ E. Rogochaya,${ }^{75}$ D. Rohr,${ }^{33}$ D. Röhrich, ${ }^{21}$ P. S. Rokita, ${ }^{142}$ F. Ronchetti, ${ }^{51}$ E. D. Rosas, ${ }^{69}$ K. Roslon, ${ }^{142}$ A. Rossi, ${ }^{28 a, 28 b, 56}$ A. Rotondi, ${ }^{139}$ A. Roy, ${ }^{49}$ P. Roy, ${ }^{109}$ O. V. Rueda,${ }^{80}$ R. Rui,${ }^{24 a, 24 b}$ B. Rumyantsev, ${ }^{75}$ A. Rustamov ${ }^{86}$ E. Ryabinkin, ${ }^{87}$ Y. Ryabov, ${ }^{97}$ A. Rybicki, ${ }^{118}$ H. Rytkonen, ${ }^{126}$ O. A. M. Saarimaki, ${ }^{43}$ S. Sadhu, ${ }^{141}$ S. Sadovsky, ${ }^{90}$ K. Šafař́k, ${ }^{36}$ S. K. Saha, ${ }^{141}$ B. Sahoo,${ }^{48}$ P. Sahoo, ${ }^{48,49}$ R. Sahoo, ${ }^{49}$ S. Sahoo, ${ }^{65}$ P. K. Sahu, ${ }^{65}$ J. Saini, ${ }^{141}$ S. Sakai, ${ }^{133}$ S. Sambyal, ${ }^{100}$ V. Samsonov, ${ }^{92,97}$ D. Sarkar,${ }^{143}$ N. Sarkar, ${ }^{141}$ P. Sarma, ${ }^{41}$ V. M. Sarti, ${ }^{104}$ M. H. P. Sas, ${ }^{63}$ E. Scapparone, ${ }^{53}$ B. Schaefer, ${ }^{95}$ J. Schambach, ${ }^{119}$ H. S. Scheid, ${ }^{68}$ C. Schiaua, ${ }^{47}$ R. Schicker, ${ }^{103}$ A. Schmah, ${ }^{103}$ C. Schmidt, ${ }^{106}$ H. R. Schmidt, ${ }^{102}$ M. O. Schmidt, ${ }^{103}$ M. Schmidt, ${ }^{102}$ N. V. Schmidt, ${ }^{68,95}$ A. R. Schmier, ${ }^{130}$ J. Schukraft, ${ }^{88}$ Y. Schutz, ${ }^{33,136}$ K. Schwarz, ${ }^{106}$ K. Schweda, ${ }^{106}$ G. Scioli, ${ }^{26 a, 26 b}$ E. Scomparin, ${ }^{58}$ M. Šefč́k,${ }^{37}$ J.E. Seger, ${ }^{15}$ Y. Sekiguchi, ${ }^{132}$ D. Sekihata, ${ }^{132}$ I. Selyuzhenkov, ${ }^{92,106}$ S. Senyukov, ${ }^{136}$ D. Serebryakov, ${ }^{62}$ E. Serradilla, ${ }^{71}$ A. Sevcenco, ${ }^{67}$ A. Shabanov, ${ }^{62}$ A. Shabetai, ${ }^{114}$ R. Shahoyan, ${ }^{33}$ W. Shaikh,${ }^{109}$ A. Shangaraev, ${ }^{90}$ A. Sharma,${ }^{99}$ A. Sharma, ${ }^{100}$ H. Sharma, ${ }^{118}$ M. Sharma, ${ }^{100}$ N. Sharma, ${ }^{99}$ A. I. Sheikh, ${ }^{141}$ K. Shigaki, ${ }^{45}$ M. Shimomura, ${ }^{82}$ S. Shirinkin, ${ }^{91}$ Q. Shou, ${ }^{39}$ Y. Sibiriak, ${ }^{87}$ S. Siddhanta, ${ }^{54}$ T. Siemiarczuk, ${ }^{84}$ D. Silvermyr, ${ }^{80}$ G. Simatovic, ${ }^{89}$ G. Simonetti,,${ }^{33,104}$ R. Singh, ${ }^{85}$ R. Singh, ${ }^{100}$ R. Singh, ${ }^{49}$ V. K. Singh, ${ }^{141}$ V. Singhal ${ }^{141}$ T. Sinha, ${ }^{109}$ B. Sitar, ${ }^{13}$ M. Sitta, ${ }^{31}$ T. B. Skaali, ${ }^{20}$ M. Slupecki, ${ }^{126}$ N. Smirnov, ${ }^{146}$ R. J. M. Snellings, ${ }^{63}$ T. W. Snellman, ${ }^{43,126}$ C. Soncco, ${ }^{111}$ J. Song, ${ }^{60,125}$ A. Songmoolnak, ${ }^{115}$ F. Soramel, ${ }^{28 a, 28 b}$ S. Sorensen, ${ }^{130}$ I. Sputowska, ${ }^{118}$ J. Stachel, ${ }^{103}$ I. Stan, ${ }^{67}$ P. Stankus, ${ }^{95}$ P. J. Steffanic, ${ }^{130}$ E. Stenlund, ${ }^{80}$ D. Stocco, ${ }^{114}$ M. M. Storetvedt,${ }^{35}$ L. D. Stritto, ${ }^{29 a, 29 b}$ A. A. P. Suaide, ${ }^{121}$ T. Sugitate, ${ }^{45}$ C. Suire, ${ }^{61}$ M. Suleymanov,${ }^{14}$ M. Suljic, ${ }^{33}$ R. Sultanov, ${ }^{91}$ M. Šumbera, ${ }^{94}$ S. Sumowidagdo, ${ }^{50}$ S. Swain, ${ }^{65}$ A. Szabo, ${ }^{13}$ I. Szarka, ${ }^{13}$ U. Tabassam,${ }^{14}$ G. Taillepied, ${ }^{134}$ J. Takahashi, ${ }^{122}$ G. J. Tambave, ${ }^{21}$ S. Tang, ${ }^{6,134}$ M. Tarhini, ${ }^{114}$ M. G. Tarzila, ${ }^{47}$ A. Tauro, ${ }^{33}$ G. Tejeda Muñoz,${ }^{44}$ A. Telesca, ${ }^{33}$ C. Terrevoli, ${ }^{125}$ D. Thakur, ${ }^{49}$ S. Thakur, ${ }^{141}$ D. Thomas, ${ }^{119}$ F. Thoresen, ${ }^{88}$ R. Tieulent, ${ }^{135}$ A. Tikhonov, ${ }^{62}$ A. R. Timmins,${ }^{125}$ A. Toia, ${ }^{68}$ N. Topilskaya,${ }^{62}$ M. Toppi ${ }^{51}$ F. Torales-Acosta, ${ }^{19}$ S. R. Torres,,${ }^{9,120}$ A. Trifiro, ${ }^{55}$ S. Tripathy, ${ }^{49}$ T. Tripathy, ${ }^{48}$ S. Trogolo, ${ }^{28 a, 28 b}$ G. Trombetta, ${ }^{32 \mathrm{a}, 32 \mathrm{~b}}$ L. Tropp, ${ }^{37}$ V. Trubnikov, ${ }^{2}$ W. H. Trzaska, ${ }^{126}$ T. P. Trzcinski, ${ }^{142}$ B. A. Trzeciak, ${ }^{63}$ T. Tsuji, ${ }^{132}$ A. Tumkin, ${ }^{108}$ R. Turrisi, ${ }^{56}$ T. S. Tveter,${ }^{20}$ K. Ullaland, ${ }^{21}$ E. N. Umaka, ${ }^{125}$ A. Uras, ${ }^{135}$ G. L. Usai, ${ }^{23 a, 23 b}$ A. Utrobicic, ${ }^{98}$ M. Vala, ${ }^{37}$ N. Valle, ${ }^{139}$ S. Vallero, ${ }^{58}$ N. van der Kolk, ${ }^{63}$ L. V. R. van Doremalen ${ }^{63}$ M. van Leeuwen, ${ }^{63}$ P. Vande Vyvre,${ }^{33}$ D. Varga ${ }^{145}$ Z. Varga,${ }^{145}$ M. Varga-Kofarago,${ }^{145}$ A. Vargas, ${ }^{44}$ M. Vasileiou, ${ }^{83}$ A. Vasiliev, ${ }^{87}$ O. Vázquez Doce, ${ }^{104,117}$ V. Vechernin, ${ }^{112}$ A. M. Veen, ${ }^{63}$ E. Vercellin, ${ }^{25 a, 25 b}$ S. Vergara Limón, ${ }^{44}$ L. Vermunt, ${ }^{63}$ R. Vernet, ${ }^{7}$ R. Vértesi, ${ }^{145}$ L. Vickovic, ${ }^{34}$ Z. Vilakazi, ${ }^{131}$ O. Villalobos Baillie, ${ }^{110}$ A. Villatoro Tello, ${ }^{44}$ G. Vino, ${ }^{52}$ A. Vinogradov, ${ }^{87}$ T. Virgili, ${ }^{29 a, 29 b}$ V. Vislavicius,${ }^{88}$ A. Vodopyanov, ${ }^{75}$ B. Volkel, ${ }^{33}$ M. A. Völkl, ${ }^{102}$ K. Voloshin, ${ }^{91}$ S. A. Voloshin, ${ }^{143}$ G. Volpe, ${ }^{32 a, 32 b}$ B. von Haller, ${ }^{33}$ I. Vorobyev, ${ }^{104}$ D. Voscek, ${ }^{116}$ J. Vrláková, ${ }^{37}$ B. Wagner, ${ }^{21}$ M. Weber, ${ }^{113}$ S. G. Weber ${ }^{144}$ A. Wegrzynek, ${ }^{33}$ D. F. Weiser, ${ }^{103}$ S. C. Wenzel, ${ }^{33}$ J. P. Wessels, ${ }^{144}$ J. Wiechula, ${ }^{68}$ J. Wikne, ${ }^{20}$ G. Wilk, ${ }^{84}$ J. Wilkinson, ${ }^{10,53}$ G. A. Willems,${ }^{33}$ E. Willsher, ${ }^{110}$ B. Windelband, ${ }^{103}$ M. Winn, ${ }^{137}$ W. E. Witt, ${ }^{130}$ Y. Wu, ${ }^{128}$ R. Xu, ${ }^{6}$ S. Yalcin, ${ }^{77}$ K. Yamakawa, ${ }^{45}$ S. Yang, ${ }^{21}$ S. Yano, ${ }^{137}$ Z. Yin, ${ }^{6}$ 
H. Yokoyama, ${ }^{63}$ I.-K. Yoo, ${ }^{17}$ J. H. Yoon, ${ }^{60}$ S. Yuan, ${ }^{21}$ A. Yuncu, ${ }^{103}$ V. Yurchenko, ${ }^{2}$ V. Zaccolo, ${ }^{24 a, 24 b}$ A. Zaman, ${ }^{14}$ C. Zampolli, ${ }^{33}$ H. J. C. Zanoli, ${ }^{63}$ N. Zardoshti, ${ }^{33}$ A. Zarochentsev, ${ }^{112}$ P. Závada ${ }^{66}$ N. Zaviyalov, ${ }^{108}$ H. Zbroszczyk ${ }^{142}$ M. Zhalov, ${ }^{97}$ S. Zhang, ${ }^{39}$ X. Zhang, ${ }^{6}$ Z. Zhang, ${ }^{6}$ V. Zherebchevskii, ${ }^{112}$ D. Zhou, ${ }^{6}$ Y. Zhou, ${ }^{88}$ Z. Zhou, ${ }^{21}$ J. Zhu, ${ }^{6,106}$ Y. Zhu, ${ }^{6}$ A. Zichichi, ${ }^{10,26 a, 26 b}$ M. B. Zimmermann, ${ }^{33}$ G. Zinovjev, ${ }^{2}$ and N. Zurlo ${ }^{140}$

(A Large Ion Collider Experiment Collaboration)

${ }^{1}$ A.I. Alikhanyan National Science Laboratory (Yerevan Physics Institute) Foundation, Yerevan, Armenia

${ }^{2}$ Bogolyubov Institute for Theoretical Physics, National Academy of Sciences of Ukraine, Kiev, Ukraine

${ }^{3 a}$ Bose Institute, Department of Physics, Kolkata, India

${ }^{3 \mathrm{~b}}$ Centre for Astroparticle Physics and Space Science (CAPSS), Kolkata, India

${ }^{4}$ Budker Institute for Nuclear Physics, Novosibirsk, Russia

${ }^{5}$ California Polytechnic State University, San Luis Obispo, California, USA

${ }^{6}$ Central China Normal University, Wuhan, China

${ }^{7}$ Centre de Calcul de l'IN2P3, Villeurbanne, Lyon, France

${ }^{8}$ Centro de Aplicaciones Tecnológicas y Desarrollo Nuclear (CEADEN), Havana, Cuba

${ }^{9}$ Centro de Investigación y de Estudios Avanzados (CINVESTAV), Mexico City and Mérida, Mexico

${ }^{10}$ Centro Fermi-Museo Storico della Fisica e Centro Studi e Ricerche "Enrico Fermi', Rome, Italy

${ }^{11}$ Chicago State University, Chicago, Illinois, USA

${ }^{12}$ China Institute of Atomic Energy, Beijing, China

${ }^{13}$ Comenius University Bratislava, Faculty of Mathematics, Physics and Informatics, Bratislava, Slovakia

${ }^{14}$ COMSATS University Islamabad, Islamabad, Pakistan

${ }^{15}$ Creighton University, Omaha, Nebraska, USA

${ }^{16}$ Department of Physics, Aligarh Muslim University, Aligarh, India

${ }^{17}$ Department of Physics, Pusan National University, Pusan, Republic of Korea

${ }^{18}$ Department of Physics, Sejong University, Seoul, Republic of Korea

${ }^{19}$ Department of Physics, University of California, Berkeley, California, USA

${ }^{20}$ Department of Physics, University of Oslo, Oslo, Norway

${ }^{21}$ Department of Physics and Technology, University of Bergen, Bergen, Norway

${ }^{22 \mathrm{a}}$ Dipartimento di Fisica dell'Università 'La Sapienza', Rome, Italy

${ }^{22 \mathrm{~b}}$ Sezione INFN, Rome, Italy

${ }^{23 a}$ Dipartimento di Fisica dell'Università, Cagliari, Italy

${ }^{23 \mathrm{~b}}$ Sezione INFN, Cagliari, Italy

${ }^{24 a}$ Dipartimento di Fisica dell'Università, Trieste, Italy

${ }^{24 \mathrm{~b}}$ Sezione INFN, Trieste, Italy

${ }^{25 a}$ Dipartimento di Fisica dell'Università, Turin, Italy

${ }^{25 \mathrm{~b}}$ Sezione INFN, Turin, Italy

${ }^{26 a}$ Dipartimento di Fisica e Astronomia dell'Università, Bologna, Italy

${ }^{26 \mathrm{~b}}$ Sezione INFN, Bologna, Italy

${ }^{27 a}$ Dipartimento di Fisica e Astronomia dell'Università, Catania, Italy

${ }^{27 \mathrm{~b}}$ Sezione INFN, Catania, Italy

${ }^{28 \mathrm{a}}$ Dipartimento di Fisica e Astronomia dell'Università, Padova, Italy

${ }^{28 \mathrm{~b}}$ Sezione INFN, Padova, Italy

${ }^{29 a}$ Dipartimento di Fisica 'E.R. Caianiello' dell'Università, Salerno, Italy

${ }^{29 b}$ Gruppo Collegato INFN, Salerno, Italy

${ }^{30}$ Dipartimento DISAT del Politecnico and Sezione INFN, Turin, Italy

${ }^{31}$ Dipartimento di Scienze e Innovazione Tecnologica dell'Università del Piemonte Orientale and INFN Sezione di Torino, Alessandria, Italy

${ }^{32 a}$ Dipartimento Interateneo di Fisica 'M. Merlin', Bari, Italy

${ }^{32 \mathrm{~b}}$ Sezione INFN, Bari, Italy

${ }^{33}$ European Organization for Nuclear Research (CERN), Geneva, Switzerland

${ }^{34}$ Faculty of Electrical Engineering, Mechanical Engineering and Naval Architecture, University of Split, Split, Croatia

${ }^{35}$ Faculty of Engineering and Science, Western Norway University of Applied Sciences, Bergen, Norway

${ }^{36}$ Faculty of Nuclear Sciences and Physical Engineering, Czech Technical University in Prague, Prague, Czech Republic

${ }^{37}$ Faculty of Science, P.J. Šafárik University, Košice, Slovakia

${ }^{38}$ Frankfurt Institute for Advanced Studies, Johann Wolfgang Goethe-Universität Frankfurt, Frankfurt, Germany

${ }^{39}$ Fudan University, Shanghai, China

${ }^{40}$ Gangneung-Wonju National University, Gangneung, Republic of Korea 
${ }^{41}$ Gauhati University, Department of Physics, Guwahati, India

${ }^{42}$ Helmholtz-Institut für Strahlen- und Kernphysik, Rheinische Friedrich-Wilhelms-Universität Bonn, Bonn, Germany

${ }^{43}$ Helsinki Institute of Physics (HIP), Helsinki, Finland

${ }^{44}$ High Energy Physics Group, Universidad Autónoma de Puebla, Puebla, Mexico

${ }^{45}$ Hiroshima University, Hiroshima, Japan

${ }^{46}$ Hochschule Worms, Zentrum für Technologietransfer und Telekommunikation (ZTT), Worms, Germany

${ }^{47}$ Horia Hulubei National Institute of Physics and Nuclear Engineering, Bucharest, Romania

${ }^{48}$ Indian Institute of Technology Bombay (IIT), Mumbai, India

${ }^{49}$ Indian Institute of Technology Indore, Indore, India

${ }^{50}$ Indonesian Institute of Sciences, Jakarta, Indonesia

${ }^{51}$ INFN, Laboratori Nazionali di Frascati, Frascati, Italy

${ }^{52}$ INFN, Sezione di Bari, Bari, Italy

${ }^{53}$ INFN, Sezione di Bologna, Bologna, Italy

${ }^{54}$ INFN, Sezione di Cagliari, Cagliari, Italy

${ }^{55}$ INFN, Sezione di Catania, Catania, Italy

${ }^{56}$ INFN, Sezione di Padova, Padova, Italy

${ }^{57}$ INFN, Sezione di Roma, Rome, Italy

${ }^{58}$ INFN, Sezione di Torino, Turin, Italy

${ }^{59}$ INFN, Sezione di Trieste, Trieste, Italy

${ }^{60}$ Inha University, Incheon, Republic of Korea

${ }^{61}$ Institut de Physique Nucléaire d'Orsay (IPNO), Institut National de Physique Nucléaire et de Physique des Particules (IN2P3/CNRS), Université de Paris-Sud, Université Paris-Saclay, Orsay, France

${ }^{62}$ Institute for Nuclear Research, Academy of Sciences, Moscow, Russia

${ }^{63}$ Institute for Subatomic Physics, Utrecht University/Nikhef, Utrecht, Netherlands

${ }^{64}$ Institute of Experimental Physics, Slovak Academy of Sciences, Košice, Slovakia

${ }^{65}$ Institute of Physics, Homi Bhabha National Institute, Bhubaneswar, India

${ }^{66}$ Institute of Physics of the Czech Academy of Sciences, Prague, Czech Republic

${ }^{67}$ Institute of Space Science (ISS), Bucharest, Romania

${ }^{68}$ Institut für Kernphysik, Johann Wolfgang Goethe-Universität Frankfurt, Frankfurt, Germany

${ }^{69}$ Instituto de Ciencias Nucleares, Universidad Nacional Autónoma de México, Mexico City, Mexico

${ }^{70}$ Instituto de Física, Universidade Federal do Rio Grande do Sul (UFRGS), Porto Alegre, Brazil

${ }^{71}$ Instituto de Física, Universidad Nacional Autónoma de México, Mexico City, Mexico

${ }^{72}$ iThemba LABS, National Research Foundation, Somerset West, South Africa

${ }^{73}$ Jeonbuk National University, Jeonju, Republic of Korea

${ }^{74}$ Johann-Wolfgang-Goethe Universität Frankfurt Institut für Informatik, Fachbereich Informatik und Mathematik, Frankfurt, Germany

${ }^{75}$ Joint Institute for Nuclear Research (JINR), Dubna, Russia

${ }^{76}$ Korea Institute of Science and Technology Information, Daejeon, Republic of Korea

${ }^{77}$ KTO Karatay University, Konya, Turkey

${ }^{78}$ Laboratoire de Physique Subatomique et de Cosmologie, Université Grenoble-Alpes, CNRS-IN2P3, Grenoble, France

${ }^{79}$ Lawrence Berkeley National Laboratory, Berkeley, California, USA

${ }^{80}$ Lund University Department of Physics, Division of Particle Physics, Lund, Sweden

${ }^{81}$ Nagasaki Institute of Applied Science, Nagasaki, Japan

${ }^{82}$ Nara Women's University (NWU), Nara, Japan

${ }^{83}$ National and Kapodistrian University of Athens, School of Science, Department of Physics, Greece

${ }^{84}$ National Centre for Nuclear Research, Warsaw, Poland

${ }^{85}$ National Institute of Science Education and Research, Homi Bhabha National Institute, Jatni, India

${ }^{86}$ National Nuclear Research Center, Baku, Azerbaijan

${ }^{87}$ National Research Centre Kurchatov Institute, Moscow, Russia

${ }^{88}$ Niels Bohr Institute, University of Copenhagen, Copenhagen, Denmark

${ }^{89}$ Nikhef, National institute for subatomic physics, Amsterdam, Netherlands

${ }^{90}$ NRC Kurchatov Institute IHEP, Protvino, Russia

${ }^{91} N R C$ «Kurchatov Institute»-ITEP, Moscow, Russia

${ }^{92}$ NRNU Moscow Engineering Physics Institute, Moscow, Russia

${ }^{93}$ Nuclear Physics Group, STFC Daresbury Laboratory, Daresbury, United Kingdom

${ }^{94}$ Nuclear Physics Institute of the Czech Academy of Sciences, $\check{R} e \check{u}$ Prahy, Czech Republic

${ }^{95}$ Oak Ridge National Laboratory, Tennessee, USA

${ }^{96}$ Ohio State University, Columbus, Ohio, USA

${ }^{97}$ Petersburg Nuclear Physics Institute, Gatchina, Russia

${ }^{98}$ Physics department, Faculty of science, University of Zagreb, Zagreb, Croatia

${ }^{99}$ Physics Department, Panjab University, Chandigarh, India 
${ }^{\mathrm{a}}$ Deceased.

${ }^{b}$ Dipartimento DET del Politecnico di Torino, Turin, Italy.

${ }^{c}$ M.V. Lomonosov Moscow State University, D.V. Skobeltsyn Institute of Nuclear, Physics, Moscow, Russia.

${ }^{\mathrm{d}}$ Department of Applied Physics, Aligarh Muslim University, Aligarh, India.

${ }^{\mathrm{e}}$ Institute of Theoretical Physics, University of Wroclaw, Poland. 